\title{
POSITIVE LINE BUNDLES ON ARITHMETIC VARIETIES
}

\author{
SHOUWU ZHANG
}

\section{INTRODUCTION}

For an arithmetic variety and a positive hermitian line bundle, in this paper, we compute the leading term of the Hilbert function of the line bundle, show the ampleness of the line bundle, and estimate the height of the variety in terms of the density of small points. In more details, our results are explained as follows.

Leading term of the Hilbert function. For an arithmetic variety $X$ which we refer to as a projective and flat integral scheme over $\operatorname{spec} \mathbb{Z}$, and for a relatively positive hermitian line bundle $\bar{L}$, the Hilbert function $\chi_{\text {sup }}\left(\Gamma\left(\bar{L}^{\otimes n}\right)\right)$ of positive integers is defined to count the volume of the lattice $\Gamma\left(L^{\otimes n}\right)$ of integral sections in the space $\Gamma\left(L_{\mathbb{R}}^{\otimes n}\right)$ of real sections with supremum norm. We want to prove that the leading term of this Hilbert function as $n \rightarrow \infty$ is given in terms of the height of $X$ in as $\S 1$. This is known as a theorem of Gillet and Soule [GS2] if $X$ has a regular generic fiber. Beside this known result, our proof uses Hironaka's theorem on resolutions of singularities and Minkowski's theorem on successive minima. By Hironaka's theorem, we may construct

(1) two sequences of hermitian line bundles $\left\{L_{n}^{\prime}\right\}$ and $\left\{L_{n}^{\prime \prime}\right\}$ on a fixed generic resolution $\tilde{X}$ of $X$, such that they are numerically close to the sequence $\left\{L^{\otimes n}\right\}$;

(2) some sequences of embeddings with small norms

$$
\Gamma\left(L_{n}^{\prime}\right) \subset \Gamma\left(L^{\otimes n}\right) \subset \Gamma\left(L_{n}^{\prime \prime}\right) .
$$

By Minkowski's theorem, we may obtain a lower (resp. upper) bound for the Hilbert function of $L$ by corresponding functions induced by $\left\{L_{n}^{\prime}\right\}$ (resp. $\left\{L_{n}^{\prime \prime}\right\}$ ). Applying the known results on $\tilde{X}$ we obtain the required estimate for $\left\{L^{\otimes n}\right\}$.

Arithmetic ampleness. For an arithmetic variety $X$ and a numerically positive hermitian line bundle $L$, we prove that the group $\Gamma\left(L^{\otimes n}\right)$ has a basis consisting of small sections when $n$ is sufficiently large in $\S 4$. We use a similar idea as in the context of algebraic geometry [Ha]. By our estimate of the leading term of the Hilbert function and by some lattice arguments, we reduce the proof to proving that, for any subvariety $Y_{\mathbb{C}}$ of $X_{\mathbb{C}}$, the map

$$
\Gamma\left(L_{\mathbb{C}}^{\otimes n}\right) \rightarrow \Gamma\left(\left.L_{\mathbb{C}}^{\otimes n}\right|_{Y_{\mathbf{C}}}\right)
$$

Received by the editors February 15, 1992 and, in revised form, December 1, 1993.

1991 Mathematics Subject Classification. Primary 14G40; Secondary 11G35. 
is "surjective" in the metric sense: given finitely many fixed sections $l_{1}, \cdots, l_{k}$, any section $l_{1}^{\alpha_{1}} \cdots l_{k}^{\alpha_{k}}\left(\alpha_{i} \geq 0\right)$ of $\left.L^{\otimes\left(\alpha_{1}+\cdots\right)}\right|_{Y}$ with $\alpha_{1}+\cdots+\alpha_{n}$ sufficiently large can be lifted to a section on $X$ with a small supremum norm. We prove this in two steps. The first step $(\S 2)$ is devoted to proving the assertion for compact complex manifolds using Hormander's $L^{2}$-estimate. The second step (§3) is devoted to proving the assertion for singular varieties, where we introduce the ampleness of the metric and work on nonarchimedean metrics at the same time.

Density of small points. We have two results under this title. The first result is an estimate of the height of an arithmetic variety in terms of the density of small points $(\S 5)$. This gives a more precise version of Kleiman's theorem on ampleness of a line bundle in terms of intersection numbers with curves, in the context of algebraic geometry. The proof of our result is similar to that of Kleiman's theorem [Ha]. One typical consequence is as follows: for an arithmetic variety $X$ and a semipositive hermitian line bundle $L$, the height of $X$ is 0 if and only if, on any nonempty Zariski open subset $U$, the height function on $U(\mathbb{Q})$ has the infimum 0 .

The second result $(\S 6)$ is as follows: a subvariety $X$ of a multiplicative group $\mathbb{G}_{m}^{n}$ is of the form $x H$, where $x$ is a torsion point and $H$ is a subgroup, if and only if small points of $X(\widetilde{\mathbb{Q}})$ are dense with respect to the usual height function $h_{\max }$. For proof, we embed $\mathbb{G}_{m}^{n}$ to $\left(\mathbb{P}^{1}\right)^{n}$ as usual. Then $h_{\max }$ is equivalent to a height function $h_{\infty}$ induced by a hermitian line bundle $\bar{O}_{\infty}(1)=$ $\left(O(1),\|\cdot\|_{\infty}\right)$. Approximating $\|\cdot\|_{\infty}$ by smooth metrics, we are reduced to proving that the height of the Zariski closure of $X$ with respect $\bar{O}_{\infty}(1)$ is positive, if $X$ cannot be written in the form $x H$. We prove this by induction on $\operatorname{dim} X$, by representing $c_{1}\left(\bar{O}_{\infty}(1)\right)$ by certain canonical sections and by the Ihara-Serre-Tate theorem [Lan].

For an arithmetic surface, the arithmetic ampleness of a positive hermitian line bundle was conjectured by L. Szpiro and was proved in [Z1]. For an arithmetic surface without bad reduction, Szpiro [Sz] obtained a relation between the positivity of the relative dualizing sheaf and the discreteness of algebraic points with respect to Néron-Tate height. Such a result has been generalized to the general case, by arithmetic ampleness, and by an admissible pairing on a curve; see [Z2]. We expect to obtain some results in higher dimensional varieties by using the results in this paper.

I learned subjects from L. Szpiro and G. Faltings and I am very grateful to them for encouragement during the preparation of this paper and for the time they spent in teaching me. I would like to thank X. Dai, P. Deligne, G. Tian, and S. Yeung for helpful conversations, and the referee for pointing out several inaccuracies in the original manuscript. The research has been supported by NSF grant DMS-9100383. I would like to thank IAS for its hospitality.

\section{Heights of aRithmetic VARIETIES}

(1.1) Let $X$ be a complex variety of dimension $d$, and let $\bar{L}=(L,\|\cdot\|)$ be a hermitian line bundle on $X$. We say that the metric on $\bar{L}$ is smooth if, for any (analytic) morphism $f$ from the disc $\mathbb{D}^{d}=\left\{z \in \mathbb{C}^{d}:|z|<1\right\}$ to $X$, the 
pull-back metric on $f^{*}(L)$ is smooth. For example, if $X$ is a subvariety of a complex manifold and $L$ is the restriction of a smoothly metrized hermitian line on the manifold, then the metric on $L$ is smooth. In this section we always assume that all hermitian line bundles we deal with have smooth metrics.

For a hermitian line bundle $\bar{L}$ on $X$, we say that $L$ is semipositive if for any morphism $f: \mathbb{D}^{d} \rightarrow X$ the curvature form $c_{1}^{\prime}\left(f^{*}(L)\right)$ is semipositive, where $c_{1}^{\prime}\left(f^{*}(L)\right)$ is a $(1,1)$-form on $\mathbb{D}^{d}$ defined to be $\frac{\partial \bar{\partial}}{\pi i} \log \|l\|$, where $l$ is an invertible section of $f^{*}(L)$ on $\mathbb{D}^{d}$.

(1.2) By an arithmetic variety $X$ of dimension $d$, we mean an integral scheme of dimension $d$ such that the structure morphism $\pi: X \rightarrow \operatorname{spec} \mathbb{Z}$ is projective and flat. A hermitian line bundle $\bar{L}=(L,\|\cdot\|)$ on $X$ is defined to be a line bundle $L$ on $X$ with a hermitian metric $\|\cdot\|$ on $L_{\mathbb{C}}=L \otimes_{\mathbb{Z}} \mathbb{C}$, the pull-back of $L$ on $X_{\mathbb{C}}=X \otimes_{\text {spec } \mathbb{Z}}$ spec $\mathbb{C}$, such that $\|\cdot\|$ is invariant under the complex conjugation of $X_{\mathbb{C}}$. We say that $\bar{L}$ is relatively semipositive if (1) $L$ is relatively semipositive: for any closed curve $C$ on any fiber of $X$ over $\operatorname{spec} \mathbb{Z}$, the degree $\operatorname{deg}\left(\left.L\right|_{C}\right)$ of $L$ on $C$ is nonnegative; and (2) $\|\cdot\|$ is semipositive: for any finite morphism $f: \mathbb{D}^{d} \rightarrow X$, the curvature form $c_{1}^{\prime}\left(f^{*} \bar{L}\right)$ is semipositive pointwise.

Let $X$ be an arithmetic variety, let $L$ be a hermitian line bundle on $X$, and let $f: \tilde{X} \rightarrow X$ be a generic resolution of singularities of $X$. This means that $f$ is a birational morphism from an arithmetic variety $\tilde{X}$ with regular generic fiber over $\operatorname{spec} \mathbb{Z}$. By the Hironaka theorem [Hi], such a resolution always exists. Then $f^{*}(L)$ is a hermitian line bundle (with smooth metric) on $\tilde{X}$, and the number $c_{1}\left(f^{*} \bar{L}\right)^{d}=\hat{c}_{1}\left(f^{*} \bar{L}\right)^{d}$ is defined as in [GS1], [F2]. One can prove that this number does not depend on the choice of $f$. In fact if $f_{i}: \tilde{X}_{i} \rightarrow X$ $(i=1,2)$ are two resolutions, then we can find a third resolution

$$
g: \tilde{X} \rightarrow \tilde{X}_{1} \times_{X} \tilde{X}_{2} .
$$

Using the projection formula, one can prove that both $c_{1}\left(f_{i}^{*} \bar{L}\right)^{d}$ coincide with $c_{1}\left(f^{*} \bar{L}\right)^{d}$, where $f$ is the canonical morphism from $\tilde{X}$ to $X$. We call this number the height of $X$ with respect to $\bar{L}$, and denote it by $c_{1}(\bar{L})^{d}$.

(1.3) The main aim of this section is to compute the leading terms of "Hilbert functions". We fix the following notation. Let $V$ be a real vector space with a norm $\|\cdot\|$, and let $\Gamma$ be a lattice of $V$. Then there is a unique invariant measure on $V$ such that the volume of the unit ball $\{v \in V:\|v\| \leq 1\}$ is 1 . We define that

$$
\chi_{\|\cdot\|}(\Gamma)=-\log \operatorname{vol}(V / \Gamma)
$$

Let $X$ be an arithmetic variety, let $\bar{L}$ be a hermitian line bundle, and let $\|\cdot\|_{\text {sup }}$ denote the supremum norm on $\Gamma\left(X_{\mathbb{R}}, L_{\mathbb{R}}\right)$ :

$$
\|l\|_{\text {sup }}=\sup _{x \in X(\mathbb{C})}\|l\|(x) \text {. }
$$

Theorem (1.4). Let $X$ be an arithmetic variety of dimension $d$, and let $L$ and $N$ be two hermitian line bundle on $X$ such that $L_{\mathbb{Q}}$ is ample and $\bar{L}$ is relatively 
semipositive. Then as $n \rightarrow \infty$, we have

$$
\chi_{\text {sup }}\left(\Gamma\left(X, \bar{L}^{\otimes n} \otimes N\right)\right)=\frac{n^{d}}{d !} c_{1}(\bar{L})^{d}+o\left(n^{d}\right) .
$$

We start from the following result of Gillet and Soulé:

Lemma (1.5). The assertion (1.4) is true if the following conditions are verified:

(1) $X$ has a regular generic fiber;

(2) $L$ is relatively ample;

(3) $c_{1}^{\prime}(\bar{L})$ is positive pointwise.

Proof. Assume conditions are verified. Let $g$ be a Kahler metric on $X_{\mathbf{C}}$ with Kahler form $c_{1}^{\prime}(\bar{L})$. Let $\Gamma\left(X_{\mathbf{R}}, \bar{L}_{\mathbf{R}}\right)_{L^{2}}$ denote the space $\Gamma\left(X_{\mathbb{R}}, L_{\mathbb{R}}\right)$ with the $L^{2}$ norm induced by $g$ on $X_{\mathrm{C}}$. By an arithmetic Riemann-Roch theorem proved by Gillet and Soulé and by an estimate of Bismut and Vasserot on analytic torsions, we have that

$$
\chi_{L^{2}}\left(\Gamma\left(X, \bar{L}^{\otimes n} \otimes N\right)\right)=\frac{n^{d}}{d !} c_{1}(\bar{L})^{d}+O\left(n^{d-1} \log n\right) .
$$

The assertion of the theorem follows from this estimate and the following inequality of Gromov: there is a constant $c>0$ such that $c^{-1}\|l\|_{L^{2}} \leq\|l\|_{\text {sup }} \leq$ $c n^{d}\|l\|_{L^{2}}$ for all $l$ in $\Gamma\left(X_{\mathbb{C}}, L_{\mathbb{C}}^{\otimes n} \otimes N\right)$. See [GS2], [F2], and [BV] for details.

Lemma (1.6). Let $f_{1}: X_{1} \rightarrow X$ and $f_{2}: X_{2} \rightarrow X$ be two projective morphisms of arithmetic varieties. Let $L_{1}, L_{2}, M$ be hermitian line bundles on $X_{1}, X_{2}, X$ respectively, with $L_{1 Q}$ and $L_{2 Q}$ ample. There is a constant $c$ such that the following condition is verified. For any $n_{1} \geq 0, n_{2} \geq 0$ there is a set of linear independent elements of maximal rank of $\Gamma\left(f_{1 *} L_{1}^{\otimes n_{1}} \otimes f_{2 *} L_{2}^{\otimes n_{2}} \otimes M\right)$ such that each element has norm $\leq c^{\max \left(n_{1}, n_{2}\right)}$.

Proof. We consider the special case that $M=O_{X}$ only; the general case follows from the same approach. Since the algebra

$$
\Gamma\left(L^{*}\right)_{\mathbb{Q}}=\bigoplus_{n_{1}, n_{2}} \Gamma\left(f_{1 *} L_{1 \mathbb{Q}}^{\otimes n_{1}} \otimes f_{2 *} L_{2 \mathbb{Q}}^{\otimes n_{2}}\right)
$$

is finitely generated over $\mathbb{Q}$, there are finitely many elements $s_{1}, \cdots, s_{k}$ of $\Gamma\left(L^{*}\right)$ of multidegree $\left(d_{1}, e_{1}\right), \cdots,\left(d_{k}, e_{k}\right)$ which generate $\Gamma\left(L^{*}\right)_{\mathbb{Q}}$. Replacing them by some integral multiple, we may assume that all of them are integral. Now for any $n_{1}>0, n_{2}>0$, the group $\Gamma\left(f_{1 *} L_{1}^{\otimes n_{1}} \otimes f_{2 *} L_{2}^{\otimes n_{2}}\right)$ contains the following set of elements of maximal rank:

$$
M_{n_{1}, n_{2}}=\left\{l_{\alpha}=\prod_{i} s_{i}^{\alpha_{i}}: \alpha_{i} \geq 0, \sum_{i} \alpha_{i} d_{i}=n_{1}, \sum_{i} \alpha_{i} e_{i}=n_{2}\right\} .
$$

Let $c=\max _{i}\left\|s_{i}\right\|_{\text {sup }}$. For each $l_{\alpha} \in M_{n_{1}, n_{2}}$, we have

$$
\left\|l_{\alpha}\right\|_{\text {sup }} \leq c^{\sum \alpha_{i}} \leq c^{\max \left(n_{1}, n_{2}\right)} .
$$

This proves the lemma. 
Lemma (1.7). Let $V$ be a real vector space of dimension $d$ with a norm $\|\cdot\|$, and let $\Gamma$ be a lattice in $V$. For each $1 \leq i \leq d$, let $\lambda_{i}(\Gamma)$ denote the smallest number $\lambda$ such that there exist $i$-independent elements of $\Gamma$ with norm $\leq \lambda$. Let $V^{\prime}$ be a subspace of $V$ of dimension $d^{\prime}$, and let $\Gamma^{\prime}$ be a lattice in $V^{\prime}$ which is contained in $\Gamma$. Then

$$
\chi_{\|\cdot\|}(\Gamma)-\chi_{\|\cdot\|}\left(\Gamma^{\prime}\right) \geq-\log (d !)-\left(d-d^{\prime}\right) \log \left(\frac{1}{2} \lambda_{d}(\Gamma)\right) .
$$

Proof. By Minkowski's theorem we have the following estimate:

$$
\frac{2^{d}}{d !} \operatorname{vol}(\Gamma) \leq \lambda_{1}(\Gamma) \cdots \lambda_{d}(\Gamma) \leq 2^{d} \operatorname{vol}(\Gamma)
$$

Since $\lambda_{i}(\Gamma) \leq \lambda_{i}\left(\Gamma^{\prime}\right)$ for $1 \leq i \leq d^{\prime}$, it follows that

$$
\begin{aligned}
\operatorname{vol}(\Gamma) & \leq \frac{d !}{2^{d}} \lambda_{1}(\Gamma) \cdots \lambda_{d}(\Gamma) \\
& \leq \frac{d !}{2^{d}} \lambda_{1}\left(\Gamma^{\prime}\right) \cdots \lambda_{d^{\prime}}\left(\Gamma^{\prime}\right) \lambda_{d}(\Gamma)^{d-d^{\prime}} \\
& \leq d ! \operatorname{vol}\left(\Gamma^{\prime}\right)\left(\frac{\lambda_{d}(\Gamma)}{2}\right)^{d-d^{\prime}} .
\end{aligned}
$$

The lemma follows by taking - log on both sides.

(1.8) Proof of (1.4). For simplicity of notation, we just consider the case that $N=O_{X}$; the general case follows from the the same approach. First of all we have the following setting:

(A) Let $f: \tilde{X} \rightarrow X$ be a generic resolution of singularities of $X$, and let $\bar{M}$ be a hermitian line bundle on $\tilde{X}$ such that $M$ is very ample and the curvature $c_{1}^{\prime}(\bar{M})$ is positive pointwise. Let $s_{1}$ be a nonzero section of $M$, and let $c_{1}$ denote its norm.

(B) Choose $n_{1}$ sufficiently large such that

$$
\Gamma\left(f^{*} L_{\mathbb{Q}}^{n_{1}} \otimes M_{\mathbb{Q}}^{-1}\right)=\Gamma\left(L_{\mathbb{Q}}^{\otimes n_{1}} \otimes f_{*}\left(M_{\mathbb{Q}}^{-1}\right)\right) \neq 0 .
$$

Since for any line bundle $B$ on $\tilde{X}$ one has $\Gamma\left(B_{\mathbb{Q}}\right)=\Gamma(B) \otimes_{\mathbb{Z}} \mathbb{Q}$, it follows that there is a nonzero section $s_{2}$ of the hermitian line bundle $f^{*} \bar{L}^{n_{1}} \otimes \bar{M}^{-1}$. Let $c_{2}$ denote the norm of $s_{2}$.

(C) For any $x \in X(\mathbb{C})$ and any function $\alpha$ on $f^{-1}(x)$, let $\|\alpha\|$ denote $\sup _{y \in f^{-1}(x)}|\alpha|(y)$. Then $f_{*}\left(O_{\tilde{X}}\right)$ becomes a metrized sheaf on $X$. Let $F$ denote the coherent sheaf $\operatorname{Hom}\left(f_{*}\left(O_{\tilde{X}}\right), O_{X}\right)$. For a sufficiently large positive number $n_{2}$, there is a nonzero section $s_{3}$ of $F_{\mathbb{Q}} \otimes L_{\mathbb{Q}}^{\otimes n_{2}}$. Replacing $s_{3}$ by $m s_{3}$, where $m$ is a positive integer, we may assume that $s_{3}$ is an integral section. Let $c_{3}$ denote the norm of $s_{3}$.

(D) Let $c_{4}$ be the constants defined in (1.6) for $(\bar{L}, \bar{M})$.

Let $n_{3}>n_{1}+n_{2}$ and $n_{4}$ be any two positive integers, and let $i$ be a nonnegative integer between 0 and $n_{3}-1$. We want to estimate $\chi_{\text {sup }}\left(\Gamma\left(L^{\otimes n_{3} n_{4}+i}\right)\right)$. 
The multiplication by $s_{1}^{n_{4}}$ gives a map

$$
\alpha: V_{1}=\Gamma\left(\bar{L}^{\otimes n_{3} n_{4}+i}\right) \rightarrow V_{2}=\Gamma\left(\bar{f}^{*} L^{\otimes n_{3} n_{4}+i} \otimes \bar{M}^{n_{4}}\right)
$$

with norm bounded by $c_{1}^{n_{4}}$, where $V_{1}$ is considered a subspace of $\Gamma\left(f^{*} \bar{L}^{\otimes n_{3} n_{4}+i}\right)$. The multiplication by $s_{2}^{n_{4}}$ gives a map

$$
\Gamma\left(f^{*} \bar{L}^{\otimes\left(n_{3}-n_{1}-n_{2}\right) n_{4}+i} \otimes \bar{M}^{\otimes n_{4}}\right) \rightarrow \Gamma\left(f^{*} \bar{L}^{\otimes\left(n_{3}-n_{2}\right) n_{4}+i}\right)
$$

with norm bounded by $c_{2}^{n_{4}}$. The multiplication by $s_{3}^{n_{4}}$ gives a map

$$
\Gamma\left(f^{*} \bar{L}^{\otimes\left(n_{3}-n_{2}\right) n_{4}+i}\right)=\Gamma\left(\bar{L}^{\otimes\left(n_{3}-n_{2}\right) n_{4}+i} \otimes f_{*}\left(O_{\tilde{X}}\right)\right) \rightarrow \Gamma\left(\bar{L}^{\otimes n_{3} n_{4}+i}\right)
$$

with norm bounded by $c_{3}^{n_{4}}$. The composition of these two morphisms gives a map

$$
\beta: V_{3}=\Gamma\left(f^{*} \bar{L}^{\otimes\left(n_{3}-n_{1}-n_{2}\right) n_{4}+i} \otimes \bar{M}^{\otimes n_{4}}\right) \rightarrow V_{1}=\Gamma\left(\bar{L}^{\otimes n_{3} n_{4}+i}\right)
$$

with norm bounded by $c_{2}^{n_{4}} c_{3}^{n_{4}}$.

Applying (1.7) to $\left(\Gamma, \Gamma^{\prime}\right)=\left(V_{2}, \alpha\left(V_{1}\right)\right)$ and $\left(\Gamma, \Gamma^{\prime}\right)=\left(V_{1}, \beta\left(V_{3}\right)\right)$, we obtain that

$$
\begin{aligned}
\chi_{\text {sup }}\left(V_{1}\right) \leq & \chi_{\text {sup }}\left(\alpha\left(V_{1}\right)\right)+n_{4} \operatorname{dim}_{\mathbb{Q}}\left(V_{1 \mathbb{Q}}\right) \log c_{1} \\
\leq & \chi_{\text {sup }}\left(V_{2}\right)+\log \operatorname{dim}_{\mathbb{Q}}\left(V_{2 \mathbb{Q}}\right) !+n_{4} \operatorname{dim}_{\mathbb{Q}}\left(V_{1 \mathbb{Q}}\right) \log c_{1} \\
& +n_{3}\left(n_{4}+1\right) \operatorname{dim}_{\mathbb{Q}} \operatorname{coker}(\alpha)_{\mathbb{Q}} \log \frac{c_{4}}{2},
\end{aligned}
$$

and

$$
\begin{aligned}
\chi_{\text {sup }}\left(V_{1}\right) \geq & \chi_{\text {sup }}\left(\beta\left(V_{3}\right)\right)-\log \operatorname{dim}_{\mathbb{Q}}\left(V_{1 \mathbb{Q}}\right) !-n_{3} n_{4} \operatorname{dim}_{\mathbb{Q}} \operatorname{coker}(\beta) \log \frac{c_{4}}{2} \\
\geq & \chi_{\text {sup }}\left(V_{3}\right)-n_{4} \operatorname{dim}_{\mathbb{Q}}\left(V_{1 \mathbb{Q}}\right) \log \left(c_{2} c_{3}\right)-\log \operatorname{dim}_{\mathbb{Q}}\left(V_{1 \mathbb{Q}}\right) ! \\
& -n_{3}\left(n_{4}+1\right) \operatorname{dim}_{\mathbb{Q}} \operatorname{coker}(\beta) \log \frac{c_{4}}{2} .
\end{aligned}
$$

By Lemma (1.5), we have the following estimate:

$$
\begin{aligned}
\chi_{\text {sup }}\left(V_{2}\right) & =\frac{n_{4}^{d}}{d !} c_{1}\left(\bar{f}^{*} L^{\otimes n_{3}} \otimes \bar{M}\right)^{d}+o_{n_{3}}\left(n_{4}^{d}\right) \\
& =\frac{\left(n_{3} n_{4}+i\right)^{d}}{d !} c_{1}(\bar{L})^{d}+O\left(n_{3}^{d-1} n_{4}^{d}\right)+o_{n_{3}}\left(n_{4}^{d}\right),
\end{aligned}
$$

where $O(x)$ denotes a quantity such that $O(x) x^{-1}$ is bounded independently on $n_{3}, n_{4}$, and $o_{n_{3}}(x)$ denotes a quantity such that, for any fixed $n_{3}$, the number $o_{n_{3}}(x) x^{-1}$ tends to 0 as $x$ tends to infinity. Similarly we have

$$
\chi_{\text {sup }}\left(V_{3}\right)=\frac{\left(n_{3} n_{4}+i\right)^{d}}{d !} c_{1}(\bar{L})^{d}+O\left(n_{3}^{d-1} n_{4}^{d}\right)+o_{n_{3}}\left(n_{4}^{d}\right) .
$$

Furthermore, by the Riemann-Roch theorem for algebraic varieties, we have for $i=1,2,3$ that

$$
\operatorname{dim}_{\mathbb{Q}}\left(V_{i \mathbb{Q}}\right)=\frac{\left(n_{3} n_{4}+i\right)^{d-1}}{(d-1) !}+O\left(n_{4}^{d-1} n_{3}^{d-2}\right)+o_{n_{3}}\left(n_{4}^{d-1}\right) .
$$


Bringing (1.8.3)-(1.8.5) to (1.8.1) and (1.8.2), we obtain that

$$
\chi_{\text {sup }}\left(\Gamma\left(\bar{L}^{n_{3} n_{4}+i}\right)\right)=\frac{\left(n_{3} n_{4}+i\right)^{d}}{d !} c_{1}(\bar{L})^{d}+O\left(n_{4}^{d} n_{3}^{d-1}\right)+o_{n_{3}}\left(n_{4}^{d}\right) .
$$

For any $\epsilon>0$, we may choose $n_{3}$ such that $O\left(n_{4}^{d} n_{3}^{d-1}\right)$ is bounded by $\frac{\epsilon}{2} n_{3}^{d} n_{4}^{d}$. Then for $n_{4}$ sufficiently large, $o_{n_{3}}\left(n_{4}^{d}\right)$ is also bounded by $\frac{\epsilon}{2} n_{3}^{d} n_{4}^{d}$. This proves that, for sufficiently large $n$,

$$
\left|\chi_{\text {sup }}\left(\Gamma\left(\bar{L}^{\otimes n}\right)\right)-\frac{n^{d}}{d !} c_{1}(\bar{L})^{d}\right| \leq \epsilon n^{d} .
$$

The theorem follows.

Applying the Minkowski theorem we obtain the following result for small sections:

Corollary (1.9). Let $X$ be an arithmetic variety, and let $L$ be a hermitian line bundle on $X$. Assume that $L_{\mathbb{Q}}$ is ample and $\bar{L}$ is relatively semipositive. Then for any $\epsilon>0$ and any $n$ sufficiently large, there is a nonzero section $l$ of $L^{\otimes n}$ such that

$$
\|l\|_{\text {sup }}=\sup _{x \in X(\mathbb{C})}\|l\|(x)<\exp \left(n \epsilon-\frac{n c_{1}(\bar{L})^{d}}{d c_{1}\left(L_{\mathbb{Q}}\right)^{d-1}}\right) .
$$

(1.10) As in [GS], we may generalize (1.4) to compute the leading term of $\chi_{\text {sup }}\left(\Gamma\left(\bar{F} \otimes S^{n} \bar{E}\right)\right)$, where $E$ and $F$ are two hermitian vector bundles on $X$ with $E_{\mathbb{Q}}$ ample and $\bar{E}$ is relatively semipositive. We omit details here.

\section{LifTING SECTIONS WITH SMALL NORMS ON COMPLEX MANIFOLDS}

(2.1) Let $X$ be a compact complex manifold of dimension $d$, and let $\bar{L}$ be a hermitian line bundle with positive curvature form $c_{1}^{\prime}(\bar{L})$; then Kodaira's theorem asserts that $L$ is ample. In particular, for any subvariety $Y$ of $X$ and for $n$ sufficiently large, the map

$$
\Gamma\left(X, L^{\otimes n}\right) \rightarrow \Gamma\left(Y, L^{\otimes n}\right)
$$

is surjective. In this section we want to prove a "metrized version" of this fact.

Theorem (2.2). Let $X, Y, \bar{L}$ be assumed as in (2.1), let $l_{1}^{\prime}, \cdots, l_{s}^{\prime}$ be sections of $\left.L\right|_{Y}$, and let $\epsilon$ be a positive number. Then for any s-tuple of nonnegative integers $\alpha=\left(\alpha_{1}, \cdots, \alpha_{s}\right)$ with $|\alpha|=\sum \alpha_{i}$ sufficiently large, there is a section $l$ of $L^{\otimes|\alpha|}$ such that

$$
l_{Y}=\prod l_{i}^{\prime \alpha_{i}}
$$

and

$$
\|l\|_{X, \text { sup }} \leq e^{|\alpha| \epsilon} \prod\left\|l_{i}^{\prime}\right\|_{Y, \text { sup }}^{\alpha_{i}}
$$

(2.3) Our proof is based on a method used by Tian [T] in the proof of the density of Fubini-Study metrics, namely Hormander's $L^{2}$-estimate. We need some notation. Let $X$ be a compact complex manifold with a Kahler metric $g$, 
and let $L$ be a hermitian line bundle on $X$. We denote by $\langle$,$\rangle the induced$ hermitian products on $C^{\infty}\left(\Omega_{X}^{*}\right)$ and on $C^{\infty}\left(L \otimes \Omega_{X}^{*}\right)$, and by $\|\cdot\|,\langle,\rangle_{L^{2}}$, and $\|\cdot\|_{L^{2}}$ the corresponding norm, $L^{2}$-product, and $L^{2}$-norm (with respect to the volume form $d x$ on $X$ induced by $g$ ) respectively. Locally near a point $p$ of $X$, we may find coordinates $z_{1}, \cdots, z_{d}$ such that

$$
g\left(\frac{\partial}{\partial z_{i}}, \frac{\partial}{\partial \bar{z}_{j}}\right)=\delta_{i, j}+O\left(z^{2}\right)
$$

If $l$ is a nonzero section of $L$ we define an endomorphism $N(L)$ of $\Omega_{X}^{0.1}$ by the matrix $\left(-\frac{\partial^{2}}{\partial z_{i} \partial \bar{z}_{j}} \log \|l\|\right)$. For a function $\psi$ on $X$, let $N(\psi)$ denote the endomorphism $N(O(\psi))=\left(-\frac{\partial^{2} \psi}{\partial z_{i} \partial \bar{z}_{j}}\right)$, where $O(\psi)$ is the trivial line bundle $O$ with metric $|\cdot| \exp (-\psi)$.

Lemma (2.4). Let $T_{X}$ be the holomorphic tangent bundle on $X$ with the hermitian metric induced by $g$. Let $c$ be a positive number such that $N\left(L \otimes \operatorname{det} T_{X}^{1.0}\right) \geq$ $c$; this means that for any point $x$ of $X$ and any element $\alpha$ in $\Omega_{X, x}^{0,1}$, the following inequality is verified:

$$
\left\langle N\left(L \otimes \operatorname{det} T_{X}\right) \alpha, \alpha\right\rangle \geq c\langle\alpha, \alpha\rangle .
$$

Then for any $w \in C^{\infty}\left(L \otimes \Omega_{X}^{1,0}\right)$ such that $\bar{\partial} w=0$, there is an element $u$ in $C^{\infty}(L)$ such that $\bar{\partial} u=w$ and $\|u\|_{L^{2}}^{2} \leq \frac{1}{c}\|w\|_{L^{2}}^{2}$.

Proof. By the Bochner-Kodaira formula, for any $\alpha$ in $C^{\infty}\left(L \otimes \Omega_{X}^{0,1}\right)$ one has the following estimate:

$$
\left\langle\Delta_{\partial} \alpha, \alpha\right\rangle_{L^{2}} \geq c\|\alpha\|_{L^{2}}^{2}
$$

see $[\mathrm{BV}]$ for details.

Let $v$ be any element in $C^{\infty}\left(L \otimes \Omega_{X}^{0,1}\right)$. Write $v=v_{1}+v_{2}$ such that $\bar{\partial} v_{1}=0$ and such that $v_{2}$ is orthogonal to $\operatorname{ker}(\bar{\partial})$. It follows that $\bar{\partial}^{*} v_{2}=0$, where $\bar{\partial}^{*}$ is the adjoint of $\bar{\partial}$ with respect to $\|\cdot\|_{L^{2}}$. Applying (2.4.1) we obtain that

$$
\begin{aligned}
\left|\langle v, w\rangle_{L^{2}}\right|^{2} & =\left|\left\langle v_{1}, w\right\rangle_{L^{2}}\right|^{2} \\
& \leq\left\|v_{1}\right\|_{L^{2}}^{2}\|w\|_{L^{2}}^{2} \leq \frac{1}{c}\|w\|_{L^{2}}^{2}\left\langle\Delta_{\partial} v_{1}, v_{1}\right\rangle \\
& =\frac{1}{c}\|w\|_{L^{2}}^{2}\left\|\bar{\partial}^{*} v_{1}\right\|_{L^{2}}^{2}=\frac{1}{c}\|w\|_{L^{2}}^{2}\left\|\bar{\partial}^{*} v\right\|_{L^{2}}^{2} .
\end{aligned}
$$

Applying the Hahn-Banach theorem to the linear functional on the $\operatorname{im}\left(\bar{\partial}^{*}\right)$ in $L^{2}(L)$ :

$$
\bar{\partial}^{*} v \rightarrow\langle v, w\rangle_{L^{2}},
$$

we obtain an element $u \in L^{2}(L)$ with $\|u\|_{L^{2}}^{2} \leq \frac{1}{c}\|w\|_{L^{2}}^{2}$, such that

$$
\left\langle\bar{\partial}^{*} v, u\right\rangle_{L^{2}}=\langle v, w\rangle_{L^{2}}
$$

for any $v$. This implies that $\bar{\partial} u=w$. Since $\Delta_{\hat{\partial}} u=\bar{\partial}^{*} w$ is smooth, it follows that $u$ is a smooth section of $L$. This completes the proof of the lemma.

We have the following formal generalization: 
Lemma (2.5). Let $(X, g)$ be a compact Kahler manifold, let $L$ be a hermitian line bundle, let $\mu$ be a measure on $X$, and let $c$ be a constant. Assume that the following conditions are verified: there is a decreasing sequence $\left\{\psi_{i}\right\}$ of smooth functions on $X$ such that $e^{-\psi_{i}} d x$ converges to $\mu$, and for each $i$,

$$
\frac{1}{2} N\left(\psi_{i}\right)+N(\bar{L})+N\left(\operatorname{det} T_{X}^{1.0}\right) \geq c .
$$

Then for any $\bar{\partial}$-closed form $w$ in $C^{\infty}\left(L \otimes \Omega_{X}^{0,1}\right)$ with $\|w\|_{L^{2}(\mu)}<\infty$, there is $a u$ in $C^{\infty}(L)$ such that $\bar{\partial} u=w$ and $\|u\|_{L^{2}(\mu)}^{2} \leq \frac{1}{c}\|w\|_{L^{2}(\mu)}^{2}$, where $\|\cdot\|_{L^{2}(\mu)}$ is the $L^{2}$ norm with respect to the measure $\mu$.

Proof. For any smooth function $\psi$ on $X$, let $\bar{L}(\psi)$ denote the line bundle $L$ with hermitian metric $\|\cdot\|_{\psi}=\|\cdot\|_{L} e^{-\psi}$. Applying (2.4) to $\bar{L}\left(\frac{1}{2} \psi_{i}\right)$, we obtain a sequence $\left\{u_{i}\right\}$ of elements in $C^{\infty}(L)$ such that $\bar{\partial} u_{i}=w$ and $\left\|u_{i}\right\|_{\psi_{i}}^{2} \leq$ $\frac{1}{c}\|w\|_{\psi_{i}}^{2}$. Write $u_{i}=u_{1}+v_{i}$, where $v_{i}$ is in $\Gamma(L)$. We claim that $\left\{v_{i}\right\}$ is a bounded subset of $\Gamma(L)$. Actually for any $\psi_{i}$, let $\|\cdot\|_{\psi_{i}}$ denote the $L^{2}$ - norm with respect to measure $e^{-\psi_{i}} d x$; then

$$
\frac{1}{c}\|w\|_{L^{2}(\mu)}^{2} \geq\left\|u_{i}\right\|_{\psi_{i}}^{2} \geq\left\|u_{i}\right\|_{\psi_{1}}^{2} \geq \frac{1}{2}\left\|v_{i}\right\|_{\psi_{1}}^{2}-\left\|u_{1}\right\|_{\psi_{1}}^{2} .
$$

Our claim follows. Since $\Gamma(L)$ is of finite dimension, replacing $\left\{v_{i}\right\}$ by a subsequence we may assume that $v_{i}$ converges to an element $v$ in $\Gamma(L)$. Let $u$ denote $u_{1}+v$; then $\bar{\partial} u=w$. Since for any $j \geq i$ we have

$$
\left\|u_{j}\right\|_{\psi_{i}}^{2} \leq\left\|u_{j}\right\|_{\psi_{j}}^{2} \leq \frac{1}{c}\|w\|_{L^{2}(\mu)}^{2},
$$

it follows that

$$
\|u\|_{\psi_{i}}^{2} \leq \frac{1}{c}\|w\|_{L^{2}(\mu)}^{2}
$$

This implies that $\|u\|_{L^{2}(\mu)}^{2} \leq \frac{1}{c}\|w\|_{L^{2}(\mu)}^{2}$. The proof of the lemma is complete.

Lemma (2.6). Let $\bar{L}$ be a hermitian line bundle on a compact complex manifold such that $c_{1}^{\prime}(\bar{L})$ is positive. Let $Y$ be a reduced subvariety of $X$, let $U$ be a neighborhood of $Y$ in $X$, and let $\epsilon$ be a positive number. Then for any $n$ sufficiently large, and any section $l_{U}$ of $L^{\otimes n}$ on $U$, there is a section $l$ of $L^{\otimes n}$ such that $l_{Y}=\left.l_{U}\right|_{Y}$ and $\|l\|_{\text {sup }} \leq e^{n \epsilon}\left\|l_{U}\right\|_{\text {sup }}$.

Proof. Let $g$ be the metric on $X$ induced by the Kahler form $c_{1}^{\prime}(\bar{L})$. Let $f: \tilde{X} \rightarrow X$ be the blow-up of $X$ at $Y$, and let $E$ denote the exceptional divisor. For sufficiently large $m$, the bundle $I_{Y} \otimes L^{\otimes m}$ is generated by global sections $V=\Gamma\left(I_{Y} \otimes L^{\otimes m}\right)$, where $I_{Y}$ is the ideal sheaf of $Y$. Let $i$ denote the canonical morphism from $\tilde{X}$ to $X \times \mathbb{P}(V)$. Then $i^{*}(O(1))=f^{*} L^{\otimes m}(-E)$. Choose a basis for $V$. This gives a Fubini-Study metric on $O(1)$ with positive curvature form. Choose a metric $\|\cdot\|_{E}$ on $O(E)$ such that $\|\cdot\|_{L}^{m}\|\cdot\|_{E}$ on $f^{*} L^{\otimes m}(-E)$ agrees with the pull-back of the Fubini-Study metric. This shows that $\left(O(-E),\|\cdot\|_{E}\right)$ has curvature no less than $-m c_{1}^{\prime}(\bar{L})$. Let $\rho$ denote the 
function $\|1\|_{E}$, where 1 is the canonical section of $O(E)$. Then $\rho$ is a distance function of $Y$. On $X-Y$ we have $N(-\log \rho)=N\left(O(E),\|\cdot\|_{E}\right)$. So $N(\log \rho)$ is bounded below by $-m N(\bar{L})$ on $X$.

One can find a decreasing sequence of smooth functions $\left\{\psi_{i}\right\}$ which converges to $\log \rho$, such that the set $\left\{N\left(\psi_{i}\right)\right\}$ is uniformly bounded below in $i$ and on $X$. Actually, let $f$ be any smooth function such that (i) $f^{\prime \prime}(x) \geq 0$ for all $x$; (ii) $f(x)=x$ for $x>0$ and $f(x)=-0.5$ for $x<-1$. Then the sequence $\left\{\psi_{i}=f(\log \rho+i)-i\right\}$ will satisfy our requirements. In fact (1) since $f$ is constant for $x<-1$ it follows that $\psi_{i}$ is defined over whole $X$; (2) since $f^{\prime} \leq 1$, it follows that the sequence $\left\{\psi_{i}\right\}$ is decreasing; (3) since $f(x)=x$ for all $x>0$, it follows that $\psi_{i}$ is convergent to $\log \rho$; (4) since $0 \leq f^{\prime} \leq 1$ and $f^{\prime \prime} \geq 0$, it follows that

$$
\begin{aligned}
N\left(\psi_{k}\right) & =\left(\frac{\partial^{2} \psi_{k}}{\partial z_{i} \partial \bar{z}_{j}}\right)=f^{\prime \prime}(\log \rho+k)\left(\frac{\partial \log \rho}{\partial z_{i}} \frac{\partial \log \rho}{\partial \bar{z}_{j}}\right)+f^{\prime}(\log \rho+i) N(\log \rho) \\
& \geq-m N(\bar{L}) .
\end{aligned}
$$

Let $c_{1}$ be a constant such that $N(\bar{L})>c_{1}$ pointwise, and let $d$ denote the dimension of $X$. It follows that for sufficiently large $n$, the following inequality holds uniformly in $i$ and on $X$ :

$$
\left(d+\frac{1}{2}\right) N\left(\psi_{i}\right)+n N(\bar{L})+N\left(\operatorname{det} T_{X}^{1,0}\right) \geq c_{1} .
$$

Let $n$ be any positive number such that (2.6.1) holds, and let $l_{U}$ be a section of $L^{\otimes n}$ on $U$. Let $\theta$ be a smooth function on $X$ which is 0 out of $U$ and which is 1 on a neighborhood $U^{\prime}$ of $Y$. Let $w$ denote $\bar{\partial}\left(\theta l_{U}\right)$. Applying (2.5) we obtain a smooth section $l^{\prime}$ (which may not be holomorphic) such that $\bar{\partial} l^{\prime}=w$ and

$$
\begin{aligned}
\int_{X}\left\|l^{\prime}\right\|^{2} \rho^{-(2 d+1)} d x \leq \frac{1}{c_{1}} \int_{X}\|w\|^{2} \rho^{-(2 d+1)} d x \\
\quad=\frac{1}{c_{1}} \int_{X}\|\bar{\partial} \theta\|^{2}\left\|l_{U}\right\|^{2} \rho^{-(2 d+1)} d x \leq \frac{\left\|l_{U}\right\|_{\text {sup }}^{2}}{c_{1}} \int_{U-U^{\prime}}\|\bar{\partial} \theta\|^{2} \rho^{-(2 d+1)} d x \\
\quad=c_{2}\left\|l_{U}\right\|_{\text {sup }}^{2},
\end{aligned}
$$

where $c_{2}$ is a positive constant.

Let $l=\theta l_{U}-l^{\prime}$. Since $\bar{\partial} l=0$, it follows that $l$ is holomorphic. Since $\int\left\|l^{\prime}\right\|^{2} \rho^{-(2 d+1)} d x$ is finite, it follows that $\left.l^{\prime}\right|_{Y}=0$, i.e., $\left.l\right|_{Y}=\left.l_{U}\right|_{Y}$. To complete the proof of the lemma, we need to estimate $\|l\|_{\text {sup }}$. We estimate $\|l\|_{L^{2}}$ first. By (2.6.2), one has

$$
\begin{aligned}
\|l\|_{L^{2}}^{2} & \leq 2\left\|\theta l_{U}\right\|_{L^{2}}^{2}+2\left\|l^{\prime}\right\|_{L^{2}}^{2} \\
& \leq 2\|\theta\|_{\text {sup }}^{2}\left\|l_{U}\right\|_{\text {sup }}^{2}+2\|\rho\|_{\text {sup }}^{2 d+1} \int\left\|l^{\prime}\right\|^{2} \rho^{-(2 d+1)} d x \leq c_{3}\left\|l_{U}\right\|_{\text {sup }}^{2},
\end{aligned}
$$

where $c_{3}$ is a positive constant. 
The lemma follows from (2.6.3) and the following inequality of Gromov:

$$
\|l\|_{\text {sup }}^{2} \leq c_{4} n^{2 d}\|l\|_{L^{2}}^{2}
$$

where $c_{4}$ is a positive constant. See [GS2] for the proof of this inequality.

(2.7) Proof of (2.2). Let $n_{0}$ be a positive integer such that all sections of $L^{\otimes n}$ on $Y$ can be extended to sections on $X$. Without loss of generality we may assume that all $l_{i}$ are nonzero. For each $s$-tuple $\beta=\left(\beta_{1}, \cdots, \beta_{s}\right)$ of nonnegative integers with $n_{0} \leq|\beta|<2 n_{0}$, let $l_{\beta}$ be a fixed section of $L^{\otimes n}$ such that $\left.l_{\beta}\right|_{Y}=\prod_{i} l_{i}^{\beta_{i}}$. Let $U$ be a neighborhood of $Y$ in $X$ such that

$$
\left\|\left.l_{\beta}\right|_{U}\right\|_{\text {sup }} \leq e^{\frac{\epsilon}{2}} \prod\left\|l_{i}^{\beta_{i}}\right\|_{\text {sup }} .
$$

Now any section $\prod l_{i}^{\prime \alpha_{i}}$ with $|\alpha| \geq 2 n_{0}$ can be written as a product $\prod_{j}\left(\prod_{k} l_{k}^{\prime \gamma_{j k}}\right)$ with $n_{0} \leq\left|\gamma_{j}\right|<2 n_{0}$, where $\gamma_{j}=\left(\gamma_{j 1}, \cdots\right)$ are $s$-tuples of nonnegative integers. Applying (2.6) to the section $\left.\prod_{j} l_{\gamma_{j}}\right|_{U}$ when $|\alpha|$ is sufficiently large, we obtain a section $l$ of $L^{\otimes|\alpha|}$ on $X$ such that $\left.l\right|_{Y}=\prod l_{i}^{\alpha_{i}}$ and

$$
\|l\|_{\text {sup }} \leq e^{\frac{|\alpha| \epsilon}{2}}\left\|\prod_{j} l_{\gamma_{j}} l_{U}\right\|_{\text {sup }} \leq e^{|\alpha| \epsilon} \prod_{i}\left\|l_{i}^{\prime}\right\|_{\text {sup }}^{\alpha_{i}} .
$$

This completes the proof of the theorem.

\section{AMPLE LINE BUNDLES WITH SEMIAMPLE METRICS}

(3.1) Let $K$ be an algebraically closed normed field as in the appendix. Let $X$ be a projective variety on $\operatorname{spec} K$, and let $L$ be a line bundle on $X$ with a continuous and bounded metric as defined in (a.2). Assume that $L$ is ample; then for sufficiently large $n$ the morphism

$$
\phi_{n}: \Gamma\left(L^{\otimes n}\right) \rightarrow L^{\otimes n}
$$

is surjective, where $\Gamma\left(L^{\otimes n}\right)$ is considered as a free vector bundle on $X$. The supremum norm on $\Gamma\left(L^{\otimes n}\right)$ induces a quotient norm on $L^{\otimes n}$, whose $n$-th root gives a norm $\|\cdot\|_{n, \Gamma}$ on $L$.

The metrized line bundle $(L,\|\cdot\|)$ is said to be a semiample metrized line bundle, if lim sup $\frac{\|\cdot\|_{n}}{\|\cdot\|^{n}}$ converges to 1 uniformly on $X$. Equivalently, for any $\epsilon>0$, there is a positive integer $n$, such that for any point $x \in X(K)$, there is a nonzero section $l$ of $L^{\otimes n}$ with $\|l\|_{\text {sup }} \leq e^{n \epsilon}\|l\|(x)$.

(3.2) We fix the following assumptions and notation:

- Let $K_{0}$ be $\mathbb{R}, \mathbb{C}$, or a complete discrete valuation field, and let $K$ denote an algebraic closure of $K_{0}$, and let $K_{s}$ denote the separable closure of $K_{0}$ in $K$.

- Let $X_{0}$ be a projective variety defined over $K_{0}$, and let $Y_{0}$ be a subvariety of $X_{0}$. Denote by $X$ the variety $X_{0} \times_{\operatorname{spec} K_{0}} \operatorname{spec} K$, and denote by $Y$ the subvariety $Y_{0} \times{ }_{\operatorname{spec} K_{0}} \operatorname{spec} K$ of $X$. 
- Let $L$ be an ample line bundle on $X_{0}$ with a $\operatorname{Gal}\left(K_{s} / K_{0}\right)$ invariant and semiample metric over $X(K)$.

- Let $M$ be a coherent sheaf on $X_{0}$ such that $\left.M\right|_{Y_{0}}$ is also torsion free. Fix bounded $\operatorname{Gal}\left(K_{s} / K_{0}\right)$ invariant metrics on $M$ and $M_{Y_{0}}$ as in (a.3).

Theorem (3.3). Let $\epsilon$ be a positive number. Let $l_{1}^{\prime}, \cdots, l_{s}^{\prime}$ be $s$ sections of $\left.L\right|_{Y_{0}}$ and $m$ a section of $\left.M\right|_{Y_{0}}$. Then for any s-tuple $\alpha=\left(\alpha_{1}, \cdots, \alpha_{s}\right)$ of nonnegative integers with $|\alpha|=\sum \alpha_{i}$ sufficiently large, there is a section $l$ of $L^{\otimes|\alpha|} \otimes M$ on $X_{0}$ such that

$$
l_{Y}=m \prod_{i} l_{i}^{\alpha_{i}}
$$

and

$$
\|l\|_{\text {sup }, X} \leq e^{|\alpha| \epsilon}\|m\|_{\text {sup }, Y} \prod_{i}\left\|l_{i}^{\prime}\right\|_{\text {sup }, Y}^{\alpha_{i}} .
$$

Proof. We denote by $P(\bar{L}, \bar{M})$ the assertion of the theorem, and denote by $P(\bar{L})$ the following assertion: Under the assumption of the theorem for any $\epsilon>0$ there is a positive integer $n$ and sections $l_{1}, \cdots, l_{s}$ of $L^{\otimes n}$ such that $\left.l_{i}\right|_{Y}=l_{i}^{\prime}{ }^{n}$ and $\left\|l_{i}\right\|_{\text {sup }} \leq e^{n \epsilon}\left\|l_{i}^{\prime}\right\|_{\text {sup }}^{n}$. We have the following principles:

(A) Let $\bar{L}_{n}=\left(L,\|\cdot\|_{n}\right)$ be a sequence of metrized line bundles such that metrics are invariant under $\operatorname{Gal}\left(K / K_{0}\right)$. If $\frac{\|\cdot\|_{n}}{\|\cdot\|^{\prime}}$ converges uniformly to 1 on $X(K)$ and $P\left(\bar{L}_{n}, M\right)$ holds for all $n$, then $P(L, M)$ holds. This is easy to check by definition.

(B) Let $N$ be any positive number. If $P\left(\bar{L}^{\otimes N}, \bar{M}\right)$ holds for all $\bar{M}$, then $P(\bar{L}, \bar{M})$ holds for all $\bar{M}$. In fact, for a fixed $\bar{M}, P\left(\bar{L}^{\otimes N}, \bar{L}^{\otimes i} \otimes \bar{M}\right) \quad(i=$ $0, \cdots, N-1)$ together imply $P(L, M)$.

(C) $P(\bar{L})$ implies $P(\bar{L}, \bar{M})$. For any $\epsilon>0$, by $P(\bar{L})$, we can find a positive integer $n$ and sections $l_{1}, \cdots, l_{s}$ of $\bar{L}^{\otimes n}$ such that $\left.l_{i}\right|_{Y}=l_{i}{ }^{n}$ and $\left\|l_{i}\right\|_{\text {sup }} \leq e^{\frac{n \epsilon}{2 s}}\left\|l_{i}^{\prime}\right\|_{\text {sup }}^{n}$. For sufficiently large $n_{0}$, and for any $(s+1)$-tuple of integers $(\beta, j)=\left(\beta_{1}, \cdots, \beta_{s}, j\right)$ with $0 \leq \beta_{i}<n$ and $1 \leq j \leq s$, there are sections $m_{\beta, j}$ of $L^{\otimes\left(n_{0} n+|\beta|\right)} \otimes M$ on $X$ such that $\left.m_{\beta, j}\right|_{Y}=m l_{j}^{\prime n n_{0}} \prod l_{i}^{\beta_{i}}$. Let $c$ denote the constant

$$
\max _{\beta, j} \log \frac{\left\|m_{\beta, j}\right\|_{\text {sup }}}{\|m\|_{\text {sup }}\left\|l_{j}^{\prime}\right\|_{\text {sup }}^{n_{n}} \prod\left\|l_{i}^{\prime}\right\|_{\text {sup }}^{\beta_{i}}} .
$$

Then any section $m \prod_{i} l_{i}^{\prime \alpha_{i}}$ with $|\alpha|$ sufficiently large can be written as $\left(m l_{j}^{\prime n n_{0}} \prod_{i} l_{i}^{\beta_{i}}\right) \prod_{i} l_{i}^{\gamma_{i} n}$ with $\beta_{i}<n$ for all $i$. Let $l=m_{\beta, j} \prod l_{i}^{\gamma_{i}}$. Then $l_{Y}=m \prod l_{i}^{\prime \alpha_{i}}$ and

$$
\|l\|_{\text {sup }} \leq e^{c+\frac{\epsilon|\alpha|}{2}}\|m\|_{\text {sup }} \prod\left\|l_{i}^{\prime}\right\|_{\text {sup }}^{\alpha_{i}}
$$

The assertion follows for $|\alpha|>2 c / \epsilon$.

(D) Let $i: X \rightarrow X^{\prime}$ be an embedding from $X$ to another projective variety $X^{\prime}$ over $\operatorname{spec} K$, and let $\bar{L}^{\prime}$ be a semiample metrized line bundle on $X^{\prime}$. If $i^{*} \bar{L}^{\prime}=\bar{L}$, then $P\left(\bar{L}^{\prime}\right)$ implies $P(\bar{L})$. This is clear by definition. 
Fix $\bar{L}$ and $\bar{M}$ as in the theorem. Since $\bar{L}$ is semiample metrized, applying (A) to $\left\{\bar{L}_{n, \Gamma}=\left(L,\|\cdot\|_{n, \Gamma}\right)\right\}$, it suffices to prove $P\left(\bar{L}_{n, \Gamma}, \bar{M}\right)$ for all $n$. Applying (B) for $N=n$ and metrized line bundles $\bar{L}_{n, \Gamma}$, we need only prove $P\left(\bar{L}_{n, \Gamma}^{\otimes n}, M\right)$ for all $n$. Applying $(\mathrm{C})$ it suffices to prove all $P\left(\bar{L}_{n}^{\otimes n}\right)$. Each $\bar{L}_{n, \Gamma}^{\otimes n}$ has the property that the quotient metric by the map

$$
\Gamma\left(\bar{L}_{n, \Gamma}^{\otimes n}\right) \rightarrow \bar{L}_{n, \Gamma}^{\otimes n}
$$

coincides with the metric $\|\cdot\|_{n, \Gamma}^{n}$. So we are reduced to proving $P(L)$ provided that $L$ is very ample and that the metric on $L$ is induced by the quotient metric via map $\alpha_{X}: V=\Gamma(\bar{L}) \rightarrow L$. Let $i: X \rightarrow \mathbb{P}(V)$ denote the embedding of $X$ to the projective space associated to $V$ induced by the morphism $V \rightarrow L$. The canonical bundle $O(1)$ has a quotient metric induced by the surjective morphism $V \rightarrow O(1)$. It is easy to see that $i^{*}(O(1))$ is isometric to $\bar{L}$. Applying (D) we are reduced to proving the following lemma.

Lemma (3.4). Let $V_{0}$ be a finite-dimensional vector space over $K_{0}$ with a $K$ norm as in (a.1). On the projective space $\mathbb{P}\left(V_{0}\right)$, let $\bar{O}(1)$ denote the line bundle $O(1)$ with the quotient metric $\|\cdot\|_{O(1)}$ via the map $V \rightarrow O(1)$, where $V$ is considered as a free vector bundle on $\mathbb{P}(V)$. Then the assertion $P(\bar{O}(1))$ is true. Proof. We consider archimedean $K$ first. By principle (A), and by approximating $\|\cdot\|$ by norms $\|\cdot\|_{n}$ on $V$ such that $\|\cdot\|_{n}$ is smooth on $V-\{0\}$ and invariant under complex conjugation if $K_{0}=\mathbb{R}$, we may assume that $\|\cdot\|$ is smooth on $V-\{0\}$. It follows that the induced metric on $\bar{O}(1)$ is smooth. We claim that $c_{1}^{\prime}(\bar{O}(1))$ is semipositive pointwise. If it is not true, then there is a point $p$ and a holomorphic vector $v$ at $p$ such that $c_{1}^{\prime}(\bar{O}(1))(i v \wedge \bar{v})<0$. In other words, there is an analytic morphism $f: \mathbb{D} \rightarrow \mathbb{P}(V)$ and an invertible section $l_{0}$ of $\bar{L}=f^{*}(\bar{O}(1))$ such that $\log \left\|l_{0}\right\|=a|z|^{2}+O\left(z^{3}\right)$, where $a$ is a positive number. Now any section of $L$ on $\mathbb{D}$ with norm 1 at 0 can be written as $l=f l_{0}$ with a holomorphic function $f$ which has norm 1 at 0 . We have the estimate

$$
\int_{0}^{1} \log \|l\|\left(\rho e^{2 \pi i \theta}\right) d \theta=\int_{0}^{1} \log \left\|l_{0}\right\|\left(\rho e^{2 \pi i \theta}\right) d \theta \geq \frac{1}{2} a \rho^{2},
$$

for $\rho>0$ sufficiently small. It follows that $\|l\|_{\text {sup }}>\|l\|(0)$. This contradicts the fact from the construction of $\|\cdot\|_{O(1)}$ that there is a nonzero section $s$ in $\Gamma(O(1))=V$ such that $\|s\|$ attains its maximal value at $p$. This proves that $c_{1}^{\prime}(\bar{O}(1))$ is semipositive. Let $\|\cdot\|^{\prime}$ be any metric on $O(1)$ with positive curvature form. For example, fixing any basis of $V_{0}$ over $K_{0}$, the induced Fubini-Study on $O(1)$ has positive curvature. Now $\|\cdot\|_{O(1)}$ is approximated by $\|\cdot\|_{n}=\|\cdot\|_{O(1)}^{1-\frac{1}{n}}\|\cdot\|^{\frac{1}{n}}$. By principle $(\mathrm{A})$, assertions $P\left(O(1),\|\cdot\|_{n}\right)$ all together imply $P(\bar{O}(1))$. But $\left(O(1),\|\cdot\|_{n}\right)$ has positive curvature, so the assertion $P\left(O(1),\|\cdot\|_{n}\right)$ follows from Theorem (2.2) if $K_{0}=\mathbb{C}$. If $K_{0}=\mathbb{R}$ and $l$ is the section of a power of $L$ chosen as in Theorem (2.2) on $X_{\mathbb{C}}$, then $\frac{1}{2}(l+\sigma l)$ is a section defined on $\mathbb{R}$, which has the same image in $Y$ as $l$, and whose norm 
is not bigger than that of $l$, where $\sigma$ denotes the complex conjugation on $X_{\mathbb{C}}$. This proves $P(\bar{O}(1))$ in the archimedean case.

It remains to consider the case that $K$ is nonarchimedean. Let $R_{K}$ denote the valuation ring of $K$. Let $\tilde{V}$ denote the set $\{v \in V,\|v\| \leq 1\}$. Then $\tilde{V}$ is a module over $R_{K}$ of rank $d=\operatorname{dim} V$, and $\|\cdot\|$ is induced by this module. Notice that $\tilde{V}$ may not be finitely generated. Let $\Phi$ denote the set of all finitely generated submodules of $\tilde{V}$ of rank $d$ which are stable under $\operatorname{Gal}\left(K_{s} / K_{0}\right)$. For each $W$ in $\Phi$, let $\|\cdot\|_{W}$ denote the norm on $V$ induced by $W$ :

$$
\|v\|_{W}=\inf _{a \in K^{*}}\left\{|a|^{-1}: a v \in W\right\}
$$

Since $\cup_{W \in \Phi} W=\tilde{V}$ and $V$ is finite dimensional, one may find a sequence $W_{n}$ in $\Phi$ such that $\|\cdot\|_{W_{n}}$ converges uniformly to $\|\cdot\|$; the induced metric on $O(1)$ by $\|\cdot\|_{W_{n}}$ therefore converges uniformly to the induced metric by $\|\cdot\|$. Applying principle (A), we may assume that $\tilde{V}$ is finitely generated. It follows that there is a finite extension $E$ of $K_{0}$ which is stable under $\operatorname{Gal}\left(K_{s} / K_{0}\right)$ and a finitely generated (so free) $R_{E}$-module $W_{E}$ such that $W_{E} \otimes_{R_{E}} R_{K}$ is isomorphic to $\tilde{V}$. Let $\tilde{\mathbb{P}}$ denote $\mathbb{P}\left(W_{E}\right)$. Then one can show that the metric $\|\cdot\|_{O(1)}$ is induced by model $(\tilde{P}, \tilde{O}(1))$, where $\tilde{O}(1)$ denotes the line bundle $O_{\tilde{P}}(1)$. Now $P(\bar{O}(1))$ follows easily: Let $Y$ be any subvariety of $\mathbb{P}=\mathbb{P}(V)$ defined over $K_{0}$. Let $\tilde{Y}$ denote the Zariski closure of $Y$ in $\tilde{\mathbb{P}}$. Then for sufficiently large $n$, the map

$$
\Gamma(\tilde{\mathbb{P}}, \tilde{O}(n)) \rightarrow \Gamma(\tilde{Y}, \tilde{O}(n))
$$

is surjective. This just means that, in the map

$$
\Gamma(\mathbb{P}, \bar{O}(n)) \rightarrow \Gamma(Y, \bar{O}(n)),
$$

the induced quotient norm agrees with the original norm on the target. Now for any section $l^{\prime}$ of $O(1)$ in $Y$ defined over $K_{0}$, we may find a section $l_{1}$ in $O(n)$ defined over $E$ such that $l_{1}$ has the image $l^{\prime n}$ on $Y$ and $\left\|l_{1}\right\|_{\text {sup } X}=\left\|l^{\prime}\right\|_{\text {sup } Y}^{n}$. Let $l$ denote the following section of $O($ nde $)$ :

$$
\prod_{\sigma \in \operatorname{Gal}\left(E / K_{0}\right)} \sigma l_{1}^{e},
$$

where $d=\left[E: K_{0}\right]$ and $e$ is the inseparable index of $K_{0}$ in $E$. Then $l$ is defined on $K_{0}$ which has image $l^{\prime d n e}$ in $Y$, and $\|l\|_{\sup X}=\left\|l^{\prime}\right\|_{\text {sup } Y}^{d n e}$. The assertion $P(O(1))$ holds. This completes the proof of the lemma.

Theorem (3.5). Let $X$ be a projective variety defined over $K$, and let $\bar{L}$ be a metrized line bundle on $X$. Assume that the following conditions are verified.

(1) If $K$ is archimedean, there is an embedding $i$ from $X$ to a compact complex manifold, and a hermitian line bundle $\bar{M}$ on $Y$ with $M$ ample and $c_{1}^{\prime}(\bar{M})$ semipositive, such that $i^{*} \bar{M}$ is isometric to $\bar{L}$.

(2) If $K$ is nonarchimedean, some positive power of $\bar{L}$ is induced by a model $(\tilde{X}, \tilde{L})$ such that $L$ is ample and $\tilde{L}$ is semipositive on the special fiber of $\tilde{X}$.

Then $L$ is semiample metrized. 
Proof. We consider the case that $K$ is archimedean first. Since the ampleness for $\bar{M}$ already implies the ampleness for $\bar{L}$. We may assume that $X=Y$ and $\bar{M}=\bar{L}$; i.e., $X$ is smooth and $c_{1}^{\prime}(\bar{L})$ is semipositive. Since $L$ is ample, there is a hermitian metric $\|\cdot\|^{\prime}$ on $L$ with positive curvature form, for example the pullback of some Fubini-Study metric of $O(1)$ bundle from some $\mathbb{P}^{n}$ by an embedding by some power of $L$. Let $\bar{L}_{n}$ denote $\left(L,\|\cdot\|_{n}\right)$, where $\|\cdot\|_{n}=$ $\|\cdot\|^{1-\frac{1}{n}}\|\cdot\|^{\prime \frac{1}{n}}$ and $\|\cdot\|$ is the metric on $\bar{L}$. Then $\bar{L}_{n}$ has positive curvature and $\|\cdot\|_{n}$ converges uniformly to $\|\cdot\|$. Since ampleness for all $\bar{L}_{n}$ will imply the ampleness for $\bar{L}$, we may assume that $\bar{L}$ has positive curvature. Let $\epsilon$ be any positive number and $p$ be any point of $X$. Applying Theorem (2.2), there is a positive integer $n_{p}$ and a nonzero section $l_{p}$ of the hermitian line bundle $L^{\otimes n_{p}}$ such that $\left\|l_{p}\right\|_{\text {sup }} \leq e^{\frac{\epsilon n_{p}}{2}}\left\|l_{p}\right\|(p)$. Let $U_{p}$ be a neighborhood of $p$ in $X$ such that for any $q$ in $U_{p}$ we have $\left\|l_{p}\right\|_{\text {sup }} \leq e^{n_{p} \epsilon}\left\|l_{p}\right\|(q)$. Since $X$ is compact, we can find $p_{1}, \cdots, p_{s}$ such that $U_{p_{1}}, \cdots, U_{p_{s}}$ cover $X$. Let $n=n_{p_{1}} \cdots n_{p_{s}}$. Then for any point $p$ in $X$, we can find a nonzero section $l$ of $L^{\otimes n}$ whose supremum norm is bounded by $e^{\epsilon n}$ times the norm of $l$ at $p$. It follows that the quotient norm induced by the map $\Gamma\left(\bar{L}^{\otimes n}\right) \rightarrow \bar{L}$ is bounded by $e^{\epsilon n}$ times the norm on $\bar{L}^{\otimes n}$. This shows that $\bar{L}$ is semiample metrized.

It remains to consider the case that $K$ is nonarchimedean. Replacing $\bar{L}$ by some positive power we may assume that it is induced by a model $(\tilde{X}, \tilde{L})$. Since $\tilde{X}$ is projective, there is an ample line bundle $\tilde{M}$ on $\tilde{X}$. For any fixed positive integer $n_{1}$, the bundle $\tilde{L}^{\otimes n_{1}} \otimes \tilde{M}$ is positive on the special fiber. By the Nakai-Moishezon theorem $\tilde{L}^{\otimes n_{1}} \otimes \tilde{M}$ is ample on the special fiber, so on $\tilde{X}$. Let $n_{0}$ be a positive integer such that $\tilde{L}^{\otimes n_{1} n_{0}} \otimes \tilde{M}^{\otimes n_{0}}$ is very ample. Let $\bar{M}$ be the metrized line bundle on $X$ induced by the model $(\tilde{X}, \tilde{M})$. Since over $\tilde{X}$ the morphism

$$
\Gamma\left(\tilde{L}^{\otimes n_{0} n_{1}} \otimes \tilde{M}^{\otimes n_{0}}\right) \rightarrow \tilde{L}^{\otimes n_{0} n_{1}} \otimes \tilde{M}^{\otimes n_{0}}
$$

is surjective, the quotient metric of the map

$$
\Gamma\left(\bar{L}^{\otimes n_{0} n_{1}} \otimes \bar{M}^{\otimes n_{0}}\right) \rightarrow \bar{L}^{\otimes n_{0} n_{1}} \otimes \bar{M}^{\otimes n_{0}}
$$

coincides with the original metric on the target bundle. In particular, for any point $p$ of $X$, there is a section $l_{p}^{\prime}$ such that $\left\|l_{p}^{\prime}\right\|_{\text {sup }}=\left\|l_{p}^{\prime}\right\|(p)$.

Since $L$ is ample, there is a positive integer $n_{2}$ such that $L^{n_{2}} \otimes M^{\otimes-1}$ is very ample. Let $s_{1}, \cdots, s_{m}$ be elements of $\Gamma\left(\tilde{L}^{\otimes n_{2}} \otimes \tilde{M}^{\otimes-1}\right)$ such that they form a basis for $\Gamma\left(L^{\otimes n_{2}} \otimes M^{\otimes-1}\right)$. Write $\operatorname{div}\left(s_{i}\right)=H_{i}+V_{i}$, where $H_{i}$ are horizontal and $V_{i}$ are vertical. Then $\cap_{i}\left|H_{i}\right|=\varnothing$. Let $c_{1}$ be a number such that $c_{1} \tilde{X}_{k}-V_{i}(1 \leq i \leq s)$ are all effective, where $\tilde{X}_{k}$ denotes the special fiber of $\tilde{X}$. Then we have

$$
\inf _{p} \max _{i}\left\|s_{i}\right\|(p) \geq \exp \left(-c_{1}\right) .
$$

Let $c_{2}$ denote the number $\log \max _{i}\left\|s_{i}\right\|_{\text {sup }}$. Now for any point $p$ on $X$, there is an $i_{0}$ such that the nonzero section $l_{p}=l_{p}^{\prime} s_{i_{0}}^{n_{0}}$ of $L^{\otimes\left(n_{1}+n_{2}\right) n_{0}}$ has the property 
that

$$
\left\|l_{p}\right\|_{\text {sup }} \leq e^{n_{0}\left(c_{2}+c_{1}\right)}\left\|l_{p}\right\|(p)
$$

For any $\epsilon>0$, fix $n_{2}, c_{1}, c_{2}$ as above. Let $N=\left(n_{1}+n_{2}\right) n_{0}$. Then for $n_{1}, n_{0}$ sufficiently large, the quotient metric of the map

$$
\Gamma\left(\bar{L}^{\otimes N}\right) \rightarrow \bar{L}^{\otimes N}
$$

is bounded by $e^{\epsilon N}$ times the original metric on $\bar{L}^{\otimes N}$. This proves that $\bar{L}$ is semiample metrized.

We conclude the section by asking the following question:

(3.6) Question. Let $X$ be a projective complex variety, and let $\bar{L}$ be a hermitian line bundle on $X$ with smooth metric. Assume that $L$ is ample and $c_{1}^{\prime}(\bar{L})$ is semipositive. Is $\bar{L}$ semiample metrized?

\section{AN ARITHMEtic NAKAI-Moishezon theOREM}

(4.1) Let $S=\{\infty, 2, \cdots$,$\} be the set of all places of \mathbb{Q}$. For each $p \in S$, let $|\cdot|_{p}$ denote the valuation on $\mathbb{Q}$ such that $|p|_{p}=p^{-1}$ if $p \neq \infty$ and $|\cdot|_{\infty}$ is the ordinary absolute valuation. Let $\mathbb{Q}_{p}$ denote the completion of $\mathbb{Q}$ under $|\cdot|_{p}$, and let $\overline{\mathbb{Q}}_{p}$ be a fixed algebraic closure of $\mathbb{Q}_{p}$.

Let $X$ be an irreducible variety defined over $\mathbb{Q}$. By an adelic metrized line bundle $\bar{L}$ we mean a line bundle $L$ on $X$ and a collection of metrics $\|\cdot\|=\left\{\|\cdot\|_{p}, p \in S\right\}$, where each $\|\cdot\|_{p}$ is a (Weil) metric on $L_{p}=L \otimes_{\mathbb{Q}} \overline{\mathbb{Q}}_{p}$ which is invariant under the Galois group $\operatorname{Gal}\left(\overline{\mathbb{Q}}_{p} / \mathbb{Q}_{p}\right)$, and such that the following conditions are verified. There is a Zariski open subset $U=\operatorname{spec} \mathbb{Z}\left[\frac{1}{n}\right]$ of $\operatorname{spec} \mathbb{Z}$, a projective model $\tilde{X}$ on $U$, and a line bundle $\tilde{L}$ on $\tilde{X}$ extending $L$, such that, on each $p \in U$, the metric $\|\cdot\|_{p}$ is induced by the model

$$
\left(\tilde{X}_{p}, \tilde{L}_{p}\right)=\left(\tilde{X} \times_{U} \operatorname{spec} \mathbb{Z}_{p}, \tilde{L} \otimes_{\mathbb{Z}\left[\frac{1}{n}\right]} \mathbb{Z}_{p}\right) .
$$

We say a metrized line bundle $\bar{L}=(L,\|\cdot\|)$ is semiample metrized, if, for each $p \in S$, the metrized line bundle $\left(L_{p},\|\cdot\|_{p}\right)$ on $X_{p}$ is semiample metrized. The main result of this section is the following theorem:

Theorem (4.2). Let $X$ be an irreducible variety defined over $\mathbb{Q}$, and let $\bar{L}, \bar{M}$ be two adelic metrized line bundles on $X$, such that $\bar{L}$ is semiample metrized. Assume that, for each irreducible subvariety $Y$ of $X$, there exist a positive integer $n$ and a nontrivial strictly effective section $l$ of $\bar{L}^{\otimes n}$ on $Y$. This means that $\|l\|_{p} \leq 1$ for $p \neq \infty$ and $\|l\|_{\infty}<1$. Let $\tilde{\Gamma}\left(X, L^{\otimes n} \otimes M\right)$ denote the subgroup of $\Gamma\left(X, L^{\otimes n} \otimes M\right)$ consisting of sections $l$ with $\|l\|_{p}<1$ for all $p \neq \infty$. Then for $n$ sufficiently large, there is a basis of $\tilde{\Gamma}\left(X, L^{\otimes n} \otimes M\right)$ consisting of strictly effective sections.

(4.3) Let $V$ be a $\mathbb{Q}$ vector space. By a global norm $\|\cdot\|$ we mean a collection $\left\{\|\cdot\|_{p}\right\}$ of norms on the collection $\left\{V_{p}=V \otimes_{\mathbb{Q}} \mathbb{Q}_{p}: p \in S\right\}$ respectively. We always assume that 
(1) $\|\cdot\|_{p}$ is nonarchimedean if $p \neq \infty$ (i.e., $\|x+y\|_{p} \leq \max \left(\|x\|_{p},\|y\|_{p}\right)$ for all $x, y$ in $V_{p}$ );

(2) there is a nonzero integer $n$ and a free module $\tilde{V}_{n}$ over $\mathbb{Z}\left[\frac{1}{n}\right]$ extending $V$ such that $\|\cdot\|_{p}$ is induced by $\tilde{V}_{n}$ for all $p$ coprime with $n$.

In this way

$$
\tilde{V}=\left\{x \in V,\|x\|_{p} \leq 1 \text { for all } p \neq \infty\right\}
$$

is a lattice of $V_{\infty}$. Usually for $p \mid n,\|\cdot\|_{p}$ does not coincide with metric $\|\cdot\|_{p}^{\prime}$ induced by module $\tilde{V} \otimes_{\mathbb{Z}} \mathbb{Z}_{p}$, but we have the following estimate:

$$
\|\cdot\|_{p} \leq\|\cdot\|_{p}^{\prime} \leq p\|\cdot\|_{p} .
$$

Let $\mu(V)$ (resp. $\lambda(V)$ ) denote the smallest number $r$ such that the ball $B(r)$ of radius $r$ contains a basis (resp. of a subset of full rank) of $\tilde{V}$. Then one has the following estimate:

$$
\lambda(V) \leq \mu(V) \leq \operatorname{dim}_{\mathbb{Q}} V \lambda(V) .
$$

See 1.7 of $[\mathrm{Z1}]$ for a proof.

With notation as in (4.2), let $\Gamma(\bar{L})$ denote the $\mathbb{Q}$-vector space $\Gamma(L)$ with global norm $\|\cdot\|=\left\{\|\cdot\|_{p}\right\}$, where $\|\cdot\|_{p}$ is the supremum norm of $L \otimes_{\mathbb{Q}} \overline{\mathbb{Q}}_{p}$ on $X\left(\mathbb{Q}_{p}\right)$. Theorem (4.2) just claims that $\mu\left(\Gamma\left(\bar{L}^{\otimes n} \otimes \bar{M}\right)\right)<1$ for $n>>0$. Since $L$ is ample, this is equivalent to $\mu\left(\Gamma\left(\bar{L}^{\otimes n} \otimes \bar{M}\right)\right)<e^{-n \epsilon}$ for some $\epsilon>0$, to $\lambda\left(\Gamma\left(\bar{L}^{\otimes n} \otimes \bar{M}\right)\right)<e^{-n \epsilon}$, and finally to $\lambda\left(\Gamma\left(\bar{L}^{\otimes n} \otimes \bar{M}\right)\right)<1$.

Lemma (4.4). Let $V$ be a $\mathbb{Q}$ vector space with a global norm $\|\cdot\|$, and let

$$
0=V_{0} \subset V_{1} \subset \cdots \subset V_{n+1}=V
$$

be a filtration for $V$. Assume that, for each $i, V_{i}$ has a global norm. It induces a quotient norm on $V_{i} / V_{i-1}$. Notice that the norm on $V_{n+1}$ and the norm on $V$ may not be same. Let $f$ be the smallest positive integer such that

(1) for all $p$ coprime with $f$ the modules

$$
\tilde{V}_{i, p}=\left\{x \in V_{i} \otimes \mathbb{Q}_{p}:\|x\|_{p} \leq 1\right\}
$$

induce the $\mathbb{Q}_{p}$ norms on $V_{i}$ for all $i$;

(2) $\tilde{V}_{i, p} \subset \tilde{V}_{i+1, p}$ for all $i$.

Let $\rho_{i, p}$ denote the norm of the map $\tilde{V}_{i, p} \rightarrow V_{p}$, and $\rho_{i}$ denote $\rho_{\infty} \prod_{p \mid f} p^{2} \rho_{i, p}$. Then

$$
\lambda(V) \leq \rho_{n+1} \lambda\left(V_{n+1} / V_{n}\right)+\sum_{i<n} \rho_{i+1} \lambda\left(V_{i+1} / V_{i}\right) \operatorname{dim}_{\mathbb{Q}}\left(V_{i+1} / V_{i}\right) .
$$

Proof. We prove the following two special cases first.

Case 1. Assume that $f=1$ and for each $i$ the norm $\|\cdot\|_{\infty}$ on $V_{i}$ is induced from $V$. In this case we have that $\rho\left(V_{i}\right)=1$. By induction on $n$, we need only prove the following inequality: $\lambda(V) \leq \lambda\left(V / V_{1}\right)+\operatorname{dim}_{\mathbb{Q}} V_{1} \lambda\left(V_{1}\right)$. Let $\operatorname{dim}_{\mathbb{Q}} V_{1}=d_{1}$ and $\operatorname{dim}_{\mathbb{Q}} V=d_{1}+d_{2}$. Choose $l_{1}^{\prime}, l_{2}^{\prime}, \cdots, l_{d_{2}}^{\prime}$ in $\tilde{V}$ such that their images $m_{1}, m_{2}, \cdots, m_{d_{2}}$ form a set of maximal rank for $\tilde{V} / \tilde{V}_{1}$, and 
$\max _{i}\left\|m_{i}\right\|_{\infty}=\lambda\left(V / V_{1}\right)$. Choose $l_{1}, l_{2}, \cdots, l_{d_{1}}$ in $\tilde{V}_{1}$ such that they form a subset of maximal rank for $\tilde{V}_{1}$ and $\max _{i}\left\|l_{i}\right\|_{\infty}=\lambda\left(\tilde{V}_{1}\right)$. Choose $l_{1}^{\prime \prime}, l_{2}^{\prime \prime}, \cdots, l_{d_{2}}^{\prime \prime}$ in $V_{\mathbb{R}}$ such that, for each $i, l_{i}^{\prime \prime}$ has image $m_{i}$ and $\left\|l_{i}^{\prime \prime}\right\|_{\infty}=\left\|m_{i}\right\|_{\infty}$. Since $l_{i}^{\prime \prime}-l_{i}^{\prime}$ are in $V_{1 \mathbb{R}}$, we have real numbers $\alpha_{i j}, 1 \leq i \leq d_{2}$ and $1 \leq j \leq d_{1}$, such that

$$
l_{i}^{\prime \prime}-l_{i}^{\prime}=\sum_{j} \alpha_{i j} l_{j}
$$

Set $l_{d_{1}+i}=l_{i}^{\prime}+\sum_{j}\left[\alpha_{i j}\right] l_{j} \in \tilde{V}$ for $1 \leq i \leq d_{2}$. Then $\left\{l_{1}, l_{2}, \cdots, l_{d_{1}+d_{2}}\right\}$ is a subset of full rank in $\tilde{V}$ and

$$
\left\|l_{d_{1}+i}\right\|_{\infty}=\left\|l_{i}^{\prime \prime}-\sum_{j}\left(\alpha_{i j}-\left[\alpha_{i j}\right]\right) l_{j}\right\|_{\infty} \leq\left\|l_{i}^{\prime \prime}\right\|_{\infty}+\sum_{j}\left\|l_{j}\right\|_{\infty} .
$$

This implies that $\lambda(V) \leq \lambda\left(V / V_{1}\right)+\operatorname{dim}_{\mathbb{Q}} V_{1} \lambda\left(V_{1}\right)$.

Case 2. Assume that $n=0$. Let $\left\{l_{i}\right\}$ be a set of maximal rank in $\tilde{V}_{1}$ with $\infty$-norm bounded by $\lambda\left(V_{1}\right)$, and let $\left\{m_{i}\right\}$ be its image in $V$. Then, for each $i,\left\|m_{i}\right\|_{p} \leq 1$ if $(p, f)=1$, and $\left\|m_{i}\right\|_{p} \leq \rho_{i p}$ otherwise. Let $n_{i p}$ denote $\left[\frac{\log \rho_{i p}}{\log p}\right]+1$, and let $m_{i}^{\prime}$ denote $m_{i} \prod_{p \mid f} p^{n_{i p}}$. The subset $\left\{m_{i}^{\prime}\right\}$ in $V$ is contained in $\tilde{V}$ with maximal rank, and the $\infty$-norms of its elements are bounded by $\rho_{\infty} \Pi_{p \mid f} p \rho_{i p} \lambda\left(V_{1}\right)$.

Now we want to prove the lemma for the general case. Let $V^{\prime}$ denote the $\mathbb{Q}$-vector space $V$ whose nonarchimedean norms are induced by $\tilde{V}$. Let $V_{i}^{\prime}$ denote $V_{i}$ with the norm induced by the subspace norm of $V^{\prime}$. Then by Case 1 we have that

$$
\lambda(V)=\lambda\left(V^{\prime}\right) \leq \lambda\left(V_{n+1}^{\prime} / V_{n}^{\prime}\right)+\sum_{i<n} \lambda\left(V_{i+1}^{\prime} / V_{i}^{\prime}\right) \operatorname{dim}_{\mathbb{Q}}\left(V_{i+1} / V_{i}\right) .
$$

We need only prove that

$$
\lambda\left(V_{i+1}^{\prime} / V_{i}^{\prime}\right) \leq \lambda\left(V_{i+1} / V_{i}\right) \rho_{i+1} .
$$

By Case 2 we need only prove that for each $i$ the norm of the map $\alpha_{i}: V_{i} \rightarrow V_{i}^{\prime}$ is bounded by $\prod_{p \mid f} p \rho_{i p}$. Let $V_{i}^{\prime \prime}$ be the space with subspace global norm induced by $V$. Then $\alpha_{i}$ is the composition of the canonical maps $\beta_{i}: V_{i} \rightarrow V_{i}^{\prime \prime}$ and $\gamma_{i}: V_{i}^{\prime \prime} \rightarrow V_{i}^{\prime}$. The assertion follows, since for each $p$, the $\mathbb{Q}_{p}$-norm of $\gamma_{i}$ is bounded by $p$.

(4.5) Proof of (4.2). By (4.3.1) we need only prove that $\lambda\left(\Gamma\left(\bar{L}^{\otimes n} \otimes \bar{M}\right)\right)<1$ for any sufficiently large $n$. We use induction on $d=\operatorname{dim} X$. If $d=0$, then $X=\operatorname{spec} K$ is the spectrum of a number field and $L, M$ are vector spaces of $K$ of dimension 1 . By the assumption there is a nontrivial strictly effective section $l$ of some positive power $\bar{L}^{\otimes n_{0}}$. Let $l_{1}, \cdots, l_{s}$ be a basis of $\tilde{L}$, where $L$ is considered as a vector space of dimension $s=[K: \mathbb{Q}]$ over $\mathbb{Q}$ with a global norm. Let $m$ be a nonzero element in $M$. For $n$ sufficiently large, the 
set $\left\{l^{n} l_{i} m: i=1, \cdots, s\right\}$ generates $\Gamma\left(L^{\otimes\left(n_{0} n+1\right)} \otimes M\right)$ and consists of strictly effective sections.

Now we may assume that $d>0$ and the theorem is true for all subvarieties of lower dimensions. Let $P(\bar{L}, \bar{M})$ denote the assertion of the theorem. It is easy to see that, for any $N>0$, the assertions $P\left(\bar{L}^{\otimes N}, \bar{L}^{\otimes i} \otimes \bar{M}\right), i=$ $0,1, \cdots, N-1$, together imply $P(\bar{L}, \bar{M})$. So in the proof of $P(L, M)$, we may replace $L$ by any fixed positive power.

Replacing $L$ by a positive power, we may assume that $L$ has a nontrivial strictly effective section $l$. Let $I$ denote the ideal sheaf $I=L^{-1} l=O(-\operatorname{div} l)$. We claim there is sequence of ideals

$$
I_{0}=I \subset I_{1} \subset \cdots \subset I_{m}=O_{X}
$$

and integral subvarities $D_{1}, \cdots, D_{m}$ such that $I_{i} / I_{i-1}$ are pushforwards of torsion free sheaves $G_{i}$ on $D_{i}$. If $I_{i-1}$ is constructed and $O_{X} / I_{i-1} \neq 0$, we construct $I_{i}$ and $D_{i}$ as follows. Since $X$ is Noetherian, there is a nonzero subsheaf $F$ of $O_{X} / I_{i-1}$ such that all subsheaves of $F$ have same support. Let $D_{i}$ denote the support of $F$. Let $n$ be the maximal positive integer such that $G_{i}=I_{D_{i}}^{n} F$ is nonzero. Then $G_{i}$ is a torsion free sheaf on $D_{i}$. The preimage $I_{i}$ in $O_{X}$ of $G_{i}$ is constructed as required. Since $O_{X}$ is Noetherian, the chain $I_{0} \subset I_{1} \subset \ldots$ will stop in finitely many steps. The claim is proved.

Let $U$ be a Zariski open subset of spec $\mathbb{Z}$ such that all metrics of $\bar{L}, \bar{M}$ on $U$ are induced by a model $(\tilde{X}, \tilde{L}, \tilde{M})$, the section $l$ is regular on $\tilde{X}$, and the sequence of $I_{i}$ 's is extended to a sequence

$$
\tilde{I}_{0}=\tilde{L}^{-1} \subset \tilde{I}_{1} \subset \cdots \subset \tilde{I}_{m}=O_{\tilde{X}}
$$

with $\tilde{G}_{i}=\tilde{I}_{i} / \tilde{I}_{i-1}$ supported on integral subvarieties $\tilde{D}_{i}$. Replacing $U$ by a smaller open subset, we may assume that $\tilde{L}$ is ample on $\tilde{X}$. For each $p$ not in $U$, put Galois invariant bounded metrics on $I_{i}$ and on $G_{i}$.

For each $N=n m+r$ with $1 \leq r \leq m$, define $D_{N}=D_{r}$. Let $\bar{L}_{N}$ denote the metrized sheaf $L^{\otimes n} \otimes M \otimes I_{r}$, let $\tilde{L}_{N}$ denote the corresponding sheaf on $\tilde{X}$, and let $V_{N}$ denote the $\mathbb{Q}$-vector space $\Gamma\left(L_{N}\right)$ with global norms. Consider the filtration of $V_{n m}=\Gamma\left(X, L^{\otimes n} \otimes M\right)$ :

$$
0=V_{0} \subset V_{1} \subset \cdots \subset V_{n m} .
$$

By Lemma (4.4), and Lemmas (4.6) and (4.7) which we will prove later,

$$
\begin{aligned}
\lambda\left(V_{n m}\right) & \leq \sum_{N \leq n m} e^{(N-n m) c_{1}+c_{2}-N c_{3}+c_{4}} \operatorname{dim}_{\mathbb{Q}}\left(V_{N} / V_{N-1}\right) \\
& \leq e^{-c_{5} n+c_{6}} \operatorname{dim}_{\mathbb{Q}}\left(V_{N}\right),
\end{aligned}
$$

where $c_{1}-c_{4}$ are positive numbers defined in the following lemmas, and $c_{5}, c_{6}$ are some positive numbers independent of $n$. By the Riemann-Roch theorem, $\operatorname{dim}_{\mathbb{Q}}\left(V_{N}\right)$ is bounded by a power of $n$, it follows that

$$
\lambda\left(\Gamma\left(\bar{L}^{\otimes n} \otimes \bar{M}\right)\right)<1
$$

for sufficiently large $n$. This will complete the proof of the theorem. 
Lemma (4.6). Let $\rho_{N}$ denote the norm of the map $V_{N} \rightarrow V_{n m}$ for $N \leq n m$ defined in Lemma (4.4). There are two positive numbers $c_{1}, c_{2}$ independent of $N$ such that

$$
\rho_{N} \leq e^{c_{1}(N-n m)+c_{2}} .
$$

Proof. Write $N=m k+r$ with $0 \leq k<n$ and $1 \leq r \leq m$. Then the map $V_{N} \rightarrow V_{n m}$ is given by the multiplication of

$$
l_{N}^{\prime}=l^{n-k} l_{r},
$$

where $l_{r}$ is the injection $I_{i} \rightarrow O_{X}$, which has finite norm by (a.4). Let $U^{\prime}$ denote $S-U-\{\infty\}$; then

$$
\left\|l_{N}^{\prime}\right\|_{p}= \begin{cases}1, & \text { for } p \in U ; \\ \left\|l_{r}\right\|_{p}, & \text { for } p \in U^{\prime} \\ \|l\|_{\infty}^{n-k}\left\|l_{r}\right\|_{\infty}, & \text { for } p=\infty\end{cases}
$$

It follows that $\rho_{N} \leq\|l\|_{\infty}^{n-k} \prod_{p \in U^{\prime}}\left\|l_{r}\right\|_{p} \leq e^{(N-n m) c_{1}+c_{2}}$ for some positive constants $c_{1}, c_{2}$ independent of $N, n$.

Lemma (4.7). There are two positive numbers $c_{3}, c_{4}$ such that, for any $N$, the following estimate holds:

$$
\lambda\left(V_{N} / V_{N-1}\right) \leq e^{-c_{3} N+c_{4}} .
$$

Proof. Since $L$ is ample and (4.2) holds for subvarieties $D_{1}, \cdots, D_{m}$, by induction, we can find a positive integer $n_{0}$ such that the following conditions are verified.

(1) The algebra

$$
A=\oplus_{1 \leq i \leq m} \oplus_{n \geq 0} \Gamma\left(D_{i}, L^{\otimes n_{0} n}\right)
$$

is generated by the group

$$
A_{1}=\oplus_{1 \leq i \leq m} \Gamma\left(D_{i}, L^{\otimes n_{0}}\right) .
$$

(2) $\lambda\left(A_{1}\right)<1$. Choose a finite subset $\left\{l_{i}\right\}$ of $A_{1}$ of maximal rank such that each element belongs to a single component and is strictly effective.

Since $M=\oplus_{1 \leq i \leq m} \oplus_{N \geq 1} \Gamma\left(D_{i}, L_{N}\right)$ is a finitely generated $A$ module, there is positive constant $n_{1}$ such that this module is generated by

$$
M_{0}=\oplus_{1 \leq i \leq m} \oplus_{1 \leq N \leq n_{1} m} \Gamma\left(D_{i}, L^{\otimes N}\right)
$$

over $A$. Choose a finite subset $\left\{m_{i}\right\}$ of maximal rank in $M_{0}$ such that each element belongs to a single component and $\left\|m_{i}\right\|_{p} \leq 1$ for all $p \neq \infty$.

For $N$ sufficiently large, from the exact sequence

$$
0 \rightarrow \Gamma\left(L_{N-1}\right) \rightarrow \Gamma\left(L_{N}\right) \rightarrow \Gamma\left(D_{N}, L_{N}\right) \rightarrow 0,
$$

we obtain an isomorphism

$$
\alpha: V_{N} / V_{N-1} \rightarrow \Gamma\left(D_{N}, L_{N}\right) .
$$


We identify these two spaces via $\alpha$ and let $\|\cdot\|=\left\{\|\cdot\|_{p}\right\}$ (resp. $\|\cdot\|_{\Gamma}$ $=\left\{\|\cdot\|_{\Gamma p}\right\}$ ) denote the global norm induced from the image (resp. the domain) of $\alpha$. Notice that the subset

$$
S_{N}=\left\{l_{j \alpha}=m_{j} \prod_{i} l_{i}^{\alpha_{i}} \in \Gamma\left(D_{N}, L_{N}\right): \alpha_{i}=0,1, \cdots\right\}
$$

generates $\Gamma\left(D_{N}, L_{N}\right)$. To estimate $\lambda\left(V_{N} / V_{N-1}\right)$ we need to estimate $\|\cdot\|_{\Gamma}$ of elements in $S_{N}$.

Case 1: $p \in U$. For any $N$ sufficiently large one has the exact sequence

$$
0 \rightarrow \Gamma\left(\tilde{X}, \tilde{L}_{N-1}\right) \rightarrow \Gamma\left(\tilde{X}, \tilde{L}_{N}\right) \rightarrow \Gamma\left(\tilde{D}_{N}, \tilde{L}_{N}\right) \rightarrow 0 .
$$

It follows that, for any $p \in U$, any $l_{j \alpha}$ in $S_{N}$ has $\|\cdot\|_{\Gamma p}$ bounded by 1 .

Case 2: $p \in U^{\prime}=S-U-\{\infty\}$. Let $\epsilon$ be any positive number. Write $N=\left(k n_{0}+s\right) m+r$ with $0 \leq s \leq n_{1}$. Applying Theorem (3.3) to ample bundle $\bar{L}^{\otimes n_{0}}$, one obtains elements $l_{j \alpha}^{\prime}$ 's in $\Gamma\left(L_{N} \otimes_{\mathbb{Q}} \mathbb{Q}_{p}\right)=V_{N} \otimes_{\mathbb{Q}} \mathbb{Q}_{p}$ such that their images in $\Gamma\left(D_{N} \times \operatorname{spec} \mathbb{Q}_{p}, L_{N} \otimes \mathbb{Q}_{p}\right)$ are $l_{j \alpha}$ 's and

$$
\left\|l_{j \alpha}^{\prime}\right\| \leq p^{k \epsilon}\left\|m_{j}\right\| \prod_{i}\left\|l_{i}\right\|^{\alpha_{i}} \leq p^{k \epsilon} .
$$

It follows that $\left\|l_{j \alpha}\right\|_{\Gamma p} \leq p^{k \epsilon}$.

Case 3: $p=\infty$. Let $c$ be a positive number such that all $l_{i}$ have $\|\cdot\|_{\infty}$ less than $e^{-c}$. Then by the same argument we obtain that $\left\|l_{j \alpha}\right\|_{\Gamma \infty} \leq e^{k \epsilon-c}$. and

Let $t_{j \alpha}$ denote $\left(\prod_{p \in U^{\prime}} p^{[k \epsilon]+1}\right) l_{j \alpha}$. Then $\left\{t_{j \alpha}\right\}$ also generates $\Gamma\left(D_{N}, L_{N}\right)$

$$
\left\|t_{j \alpha}\right\|_{\Gamma p}= \begin{cases}1, & p \neq \infty \\ e^{k(\epsilon-c)} \prod_{p \in U^{\prime}} p^{[k \epsilon]+1}, & p=\infty\end{cases}
$$

It follows that, for sufficiently large $N$,

$$
\lambda\left(V_{N} / V_{N-1}\right) \leq e^{-k c+k c^{\prime} \epsilon},
$$

where $c^{\prime}$ is a positive number independent of $\epsilon, k$. Choosing a sufficiently small $\epsilon$ we may find positive constants $c_{3}$ and $c_{4}$ such that, for all $N \geq 0$,

$$
\lambda\left(V_{N} / V_{N-1}\right) \leq e^{-N c_{3}+c_{4}} .
$$

The proof of the lemma is complete.

Corollary (4.8). Let $X$ be an arithmetic variety with regular generic fiber and let $\bar{L}, \bar{M}$ be two hermitian line bundles on $X$. Assume that the following conditions are verified:

(1) $L_{\mathbb{Q}}$ is ample and $\bar{L}$ is relatively semipositive.

(2) For any irreducible horizontal subvariety $Y$ (i.e., $Y$ is flat over $\operatorname{spec} \mathbb{Z}$ ), the height $c_{1}\left(\left.\bar{L}\right|_{Y}\right)^{\operatorname{dim} Y}$ of $Y$ is positive.

Then for $n$ sufficiently large, there exists a basis of $\Gamma\left(X, L^{\otimes n} \otimes M\right)$ consisting of strictly effective sections.

Proof. By (3.5), the globally metrized line bundle $\left(L_{\mathbb{Q}},\left\{\|\cdot\|_{p}\right\}\right)$ on $X_{\mathbb{Q}}$ induced by $\bar{L}$ is semiample metrized. By (1.9), for any $Y$, some positive power of $\left.L\right|_{Y}$ 
will have a nontrivial strictly effective section. The assertion of lemma follows from (4.2).

\section{Algebraic points With SMAll heights}

(5.1) Let $X$ be an arithmetic variety of dimension $d$. Let $\bar{L}$ be a hermitian line bundle on $X$. We say $\bar{L}$ is relatively semiample if $L$ is relatively semipositive and the metric of $\bar{L}$ is semiample. By (3.5), $\bar{L}$ is relatively semiample if and only if the induced adelic metric of $L$ is semiample metrized. Modulo a positive answer to question (3.6), $\bar{L}$ is relatively semiample if and only it is relatively semipositive.

For any $x \in X(\mathbb{Q})$, let $D_{x}$ denote the Zariski closure of $x$ in $X$; then the height $h_{L}(x)$ is defined to be $\operatorname{deg}\left(\left.\bar{L}\right|_{D_{x}}\right) / \operatorname{deg}\left(D_{x}\right)$. Assume that $L_{\mathbb{Q}}$ is ample. For each subset $U$ of $X_{\mathbb{Q}}$, let $e_{L}(U)^{x}$ denote the number $\inf _{x \in U} h_{L}(x)$. For each $1 \leq i \leq d$, let

$$
e_{i}(\bar{L})=\liminf \left\{e_{L}(X-Y): Y \subset X_{\mathbb{Q}} \text { closed of codimension } i\right\} .
$$

It is clear that $e_{1}(\bar{L}) \geq e_{2}(\bar{L}) \geq \cdots \geq e_{d}(\bar{L})$. The main result of this section is the following theorem:

Theorem (5.2). Let $X$ be an arithmetic variety of dimension $d$, and let $\bar{L}$ be a hermitian line bundle such that $L_{\mathbb{Q}}$ is ample and $\bar{L}$ is relatively semiample with smooth metric. Then

$$
d e_{1}(\bar{L}) \geq \frac{c_{1}(\bar{L})^{d}}{c_{1}\left(\bar{L}_{\mathbb{Q}}\right)^{d-1}} \geq e_{1}(\bar{L})+\cdots+e_{d}(\bar{L}) .
$$

Lemma (5.3). Let $X$ be an arithmetic variety of dimension $d$, and let $\bar{M}$ be a hermitian line bundle on $X$ such that $M_{\mathbb{Q}}$ is ample and $\bar{M}$ is relative semipositive. Assume that $\Gamma(M)$ has nontrivial elements $s_{1}, \cdots, s_{d}$ such that $\cap_{i}\left|\operatorname{div}\left(s_{i}\right)\right|=\varnothing$ and $\left\|s_{i}\right\|<1$. Then we have the following assertions.

(1) $c_{1}(\bar{M})^{d}>0$.

(2) For each $1 \leq i \leq d$, there is an effective cycle $Z_{i}$ of codimension $i$ such that for any relatively semipositive hermitian line bundle $\bar{L}$, the following inequality holds:

$$
c_{1}(\bar{L})^{d-i} c_{1}(\bar{M})^{i} \geq c_{1}\left(\left.\bar{L}\right|_{Z_{i}}\right)^{d-i}
$$

(3) For any finitely many irreducible subvarieties $V_{1}, \cdots, V_{m}$ of $X_{\mathbb{Q}}$, there is a section $l$ of a positive power $M^{\otimes p}$, such that $\|l\|<1$ and $\left.l\right|_{V_{i}} \neq 0$ for all $i$.

Proof. For each $1 \leq i \leq d$, we claim that $c_{1}(\bar{M})^{i}$ can be represented by a "strictly effective" arithmetic cycle $\left(Z_{i}, g_{i}\right)$; this means that $Z_{i}$ is an effective algebraic cycle on $X$, and

$$
g_{i}=\sum_{k=0}^{i-1} f_{i k} \delta_{Z_{i k}} c_{1}^{\prime}(\bar{M})^{i-1-k},
$$


where $f_{i k}>0$ are functions on $X$ and $Z_{i k}$ are effective cycles on $X_{\mathbb{C}}$ of codimension $k$. We prove this claim by induction on $i$. If $i=1$ then $c_{1}(\bar{M})$ is represented by $\left(\operatorname{div} s_{1},-\log \left\|s_{1}\right\|\right)$. Now assume that $i>1$ and $c_{1}(\bar{M})^{i-1}$ is represented by $\left(Z_{i-1}, g_{i-1}\right)$ as required. Assume $Z_{i-1}=\sum C_{j}$ with $C_{j}$ irreducible. Since sections $s_{1}, \cdots, s_{n}$ do not have base point, for each $C_{j}$ there is a section $s_{j}^{\prime}$ in $\left\{s_{i}\right\}$ such that $\left.s_{j}^{\prime}\right|_{C_{j}} \neq 0$; then $c_{1}(\bar{M})^{i}$ is represented by

$$
\left(\sum \operatorname{div}\left(\left.s_{j}^{\prime}\right|_{C_{j}}\right), g_{i-1} c_{1}^{\prime}(\bar{M})-\sum_{j} \log \left\|s_{j}^{\prime}\right\| \delta_{C_{j}}\right) \text {. }
$$

This proves our claim. It follows that

$$
c_{1}(\bar{M})^{d}=\operatorname{deg}\left(Z_{d}, g_{d}\right) \geq \int f_{d 0} c_{1}^{\prime}(\bar{M})^{d-1}>0
$$

and

$$
c_{1}(\bar{L})^{d-i} c_{1}(\bar{M})^{i}=c_{1}\left(\left.\bar{L}\right|_{Z_{i}}\right)^{d-i}+\int g_{i} c_{1}^{\prime}(\bar{L})^{d-i} \geq c_{1}\left(\left.\bar{L}\right|_{Z_{i}}\right)^{d-i}
$$

It remains to prove (3). Replacing $\left\{V_{1}, V_{2}, \cdots, V_{m}\right\}$ by the subset of maximal elements, we may assume that $V_{i} \not \subset V_{j}$ for $i \neq j$. For each $i$ there is a section $s_{i}^{\prime}$ in $\left\{s_{1}, \cdots, s_{n}\right\}$ such that $\left.s_{i}^{\prime}\right|_{V_{i}} \neq 0$. Since $M_{\mathbb{Q}}$ is ample, there are $m$ sections $t_{1}, \cdots, t_{m}$ of some positive power $M^{\otimes p_{0}}$ such that $\left.t_{i}\right|_{V_{j}}=0$ for $i \neq j$ and $\left.t_{i}\right|_{V_{i}} \neq 0$. It follows that, for sufficiently large $p$, the section

$$
l=\sum s_{i}^{\prime p-p_{0}} t_{i}
$$

will satisfy our requirement.

Lemma (5.4). Let $(X, \bar{L})$ be assumed as in (5.2). Assume in addition that $e_{d}(\bar{L}) \geq 0$; i.e., $h_{L}(x) \geq 0$ for any $x \in X(\overline{\mathbb{Q}})$. Then $c_{1}(\bar{L})^{d} \geq 0$.

Proof. Our argument is adapted from [Ha], Chapter I, $\S 6$. By induction on $d=\operatorname{dim} X$, we may assume that $c_{1}\left(\left.\bar{L}\right|_{Y}\right)^{\operatorname{dim} Y} \geq 0$ for any subvariety $Y$ of $X$ with $\operatorname{dim} Y<d$. Let $M$ be a very ample line bundle, and let $s_{1}, s_{2}, \cdots, s_{k}$ be a basis for $\Gamma(M)$. We define the $\frac{1}{2}$-scaled Fubini-Study metric $\|\cdot\|$ as follows. For any point $x$ of $X_{\mathbb{C}}$ and any local section $s$ of $M$ with $s(x) \neq 0$, then

$$
\|s\|(x)=\frac{1}{2}\left(\sum_{i}\left|\frac{s_{i}}{s}\right|^{2}\right)^{-\frac{1}{2}}
$$

It is easy to see that $\bar{M}=(M,\|\cdot\|)$ is semiample metrized and $\left\|s_{i}\right\|_{\text {sup }} \leq \frac{1}{2}$.

For any subvariety $Y$ of $X$ which is flat over spec $\mathbb{Z}$ and is of dimension $s$, let $m_{Y i}=c_{1}\left(\left.\bar{L}\right|_{Y}\right)^{s-i} c_{1}\left(\left.\bar{M}\right|_{Y}\right)^{i}$ for $0 \leq i \leq s$. By (5.3) and assumptions, $m_{Y s}>0$, and $m_{Y i} \geq 0$ if $s<d$ or $i>0$. We need to show that $m_{X 0} \geq 0$. Let $p_{Y}(t)$ denote the polynomial

$$
\left(c_{1}\left(\left.\bar{L}\right|_{Y}\right)+t c_{1}\left(\left.\bar{M}\right|_{Y}\right)\right)^{s}=m_{Y 0}+s m_{Y 1} t+\cdots+m_{Y s} t^{s}
$$


We claim that if $t>0$ and $p_{X}(t)>0$, then $p_{X}(t) \geq t^{d} m_{X d}$. By this claim $p_{X}(t)$ does not have positive root. It follows that $m_{X 0}=p_{X}(0) \geq 0$. By continuity we need only prove our claim for $t=\frac{b}{a}$, a rational number with positive integers $a, b$, such that $p_{X}(t)>0$.

Let $\bar{L}^{\prime}$ denote the line bundle $\bar{L}^{\otimes a} \otimes \bar{M}^{\otimes b}$. Then $\bar{L}^{\prime}$ has positive height for any irreducible subvariety $Y$ which is flat over $\operatorname{spec} \mathbb{Z}$. In fact, if $Y=$ $X$, then $c_{1}\left(\bar{L}^{\prime}\right)^{d}=a^{d} p_{X}\left(\frac{b}{a}\right)>0$, and if $s=\operatorname{dim} Y<d$, then $c_{1}\left(\left.\bar{L}^{\prime}\right|_{Y}\right)^{s} \geq$ $b^{s} c_{1}\left(\left.\bar{M}\right|_{Y}\right)^{s}>0$. By (1.9) and (4.2) for $n>>0, \tilde{\Gamma}\left(X, L_{\mathbb{Q}}{ }^{1 \otimes n}\right)=\Gamma\left(X, L^{\prime \otimes n}\right)$ will have a basis $s_{1}, \cdots, s_{k}$ such that $\left\|s_{i}\right\|<1$ for all $i$. Since $L^{\prime}$ is ample, for $n>>0, \cap_{i}\left|\operatorname{div} s_{i}\right|=\varnothing$. By (5.3) and the assumptions on $\bar{L}$, we have that $c_{1}(\bar{L}) c_{1}\left(\bar{L}^{\prime}\right)^{d-1} \geq 0$. It follows that

$$
\begin{aligned}
p_{X}\left(\frac{b}{a}\right) & =a^{-d}\left(a c_{1}(\bar{L})+b c_{1}(\bar{M})\right) c_{1}\left(\bar{L}^{\prime}\right)^{d-1} \\
& \geq b a^{-d} c_{1}(\bar{M}) c_{1}\left(\bar{L}^{\prime}\right)^{d-1} \geq\left(\frac{b}{a}\right)^{d} c_{1}(\bar{M})^{d} .
\end{aligned}
$$

This proves our claim, and therefore the lemma.

(5.6) Proof of (5.2). Fix a hermitian line bundle $\bar{N}=(N,\|\cdot\|)$ such that $N$ is ample and $\|\cdot\|$ is semiample. Since numerically $\frac{1}{n} c_{1}\left(\bar{L}^{\otimes n} \otimes \bar{N}\right) \rightarrow c_{1}(\bar{L})$ as $n \rightarrow \infty$, and every term in (5.2) depends continuously on $c_{1}(\bar{L})$, it is suffices to prove (5.2) for bundle $\bar{L}^{\otimes n} \otimes \bar{N}$ for all $n>0$. In other words we may assume that $L$ is ample in the following proof.

The first inequality of (5.2) follows from (1.9) as following. Let $\epsilon$ be any positive number, and let $\bar{L}^{\prime}$ denote the hermitian line bundle

$$
\bar{L}\left(-\frac{c_{1}(\bar{L})^{d}}{d c_{1}\left(L_{\mathbb{Q}}\right)^{d-1}}+\epsilon\right),
$$

where $\bar{L}(a)=\left(L,\|\cdot\|_{L} e^{-a}\right)$ for a constant $a$. It is easy to see that $c_{1}\left(\bar{L}^{\prime}\right)^{d}>0$. By (1.9), there is a nontrivial section $s$ of a positive power $\bar{L}^{\prime \otimes n}$ with $\|s\|<1$. It follows that $L^{\prime \otimes n}$ has nonegative height at each point out of $Y=\operatorname{div}(s)$, and so does $\bar{L}^{\prime}$. Therefore

$$
e_{1}(\bar{L}) \geq \inf _{x \notin Y(\mathbb{Q})} h_{L}(x)=\inf _{x \notin Y(\mathbb{Q})} h_{L^{\prime}}(x)+\frac{c_{1}(\bar{L})^{d}}{d c_{1}\left(L_{\mathbb{Q}}\right)^{d-1}}-\epsilon \geq \frac{c_{1}(\bar{L})^{d}}{d c_{1}\left(L_{\mathbb{Q}}\right)^{d-1}}-\epsilon .
$$

Since $\epsilon$ is arbitrary, this shows the required inequality.

We use induction on $d=\operatorname{dim} X$ to prove the second inequality of (5.2). If $d=0$ it is trivial. We assume that $d>0$, and the inequality is true for any subvariety $Y$ of dimension $<d$. Let $\epsilon$ be any positive number. Consider the line bundle $\bar{M}=\bar{L}\left(-e_{d}(\bar{L})+\epsilon\right)$. Since $\bar{L}\left(-e_{d}(\bar{L})\right)$ has nonnegative height at any point of $X$, it has nonnegative height at each irreducible subvariety of $X$ by (5.4). It follows that $\bar{M}$ has positive height at each subvariety of $X$. By (4.2) some positive power $\bar{M}^{\otimes n}$ will have a basis $s_{1}, \cdots, s_{k}$ such that $\left\|s_{i}\right\|<1$ for all $i$. Since $L$ is ample, it follows that $\cap\left|\operatorname{div} s_{i}\right|=\varnothing$ for $n>>0$. 
For each $l$, by definition, there is a subvariety $Z_{i}$ (may not be irreducible) of $X_{\mathbb{Q}}$ of codimension $i$ such that

$$
e_{i}(\bar{L}) \leq \inf _{x \notin Z_{i}(\mathbb{Q})} h_{L}(x)+\epsilon .
$$

By (5.3) we may find a section $l$ of a power $\bar{M}^{\otimes m}$ such that $l$ is not zero at each generic point of each $Z_{i}$. Let $Y=\operatorname{div}(l)$. It follows that

$$
c_{1}(\bar{L})^{d-1} c_{1}(\bar{M})=\frac{1}{m} c_{1}(\bar{L})^{d-1}(Y,-\log |l|) \geq \frac{1}{m} c_{1}\left(\left.\bar{L}\right|_{Y}\right)^{d-1} .
$$

Since $m c_{1}\left(L_{\mathbb{Q}}\right)^{d-1}=c_{1}\left(\left.L_{\mathbb{Q}}\right|_{Y_{\mathbb{Q}}}\right)^{d-2}$, it follows that

$$
\frac{c_{1}(\bar{L})^{d}}{c_{1}\left(L_{\mathbb{Q}}\right)^{d-1}} \geq \frac{c_{1}\left(\left.\bar{L}\right|_{Y}\right)^{d-1}}{c_{1}\left(\left.L_{\mathbb{Q}}\right|_{Y_{\mathbb{Q}}}\right)^{d-2}}-\epsilon+e_{d}(\bar{L}) \text {. }
$$

By induction,

$$
\frac{c_{1}\left(\left.\bar{L}\right|_{Y}\right)^{d-1}}{c_{1}\left(\left.L_{\mathbf{Q}}\right|_{Y_{\mathbf{Q}}}\right)^{d-2}} \geq e_{1}\left(\left.\bar{L}\right|_{Y}\right)+e_{2}\left(\left.\bar{L}\right|_{Y}\right)+\cdots+e_{d-1}\left(\left.\bar{L}\right|_{Y}\right),
$$

and definitions,

$$
e_{i}\left(\left.\bar{L}\right|_{Y}\right) \geq \inf _{x \notin Z_{i} \cap Y} h_{L}(x) \geq e_{i}(\bar{L})-\epsilon,
$$

we have that

$$
\frac{c_{1}(\bar{L})^{d}}{c_{1}\left(L_{\mathbb{Q}}\right)^{d-1}} \geq e_{1}(\bar{L})+\cdots+e_{d}(\bar{L})-d \epsilon .
$$

Since $\epsilon$ is arbitrary, we obtain the required inequality.

Corollary (5.7). Let $X$ be an arithmetic variety of dimension $d$, and let $\bar{L}$ be a hermitian.line bundle such that $L_{\mathbb{Q}}$ is ample and $\bar{L}$ is relatively semiample with smooth metric. Then we have the following assertions:

(1) Assume that $\bar{L}$ has nonnegative height at each point of $X(\bar{Q})$. The following conditions are equivalent:

(i) Some positive power of $\bar{L}$ has a nontrivial strictly effective section.

(ii) There is a nonempty Zariski open subset $U$ of $X$ such the height function $h_{L}$ has a positive lower bound on $U$.

(iii) The height of $X$ with respect to $\bar{L}$ is positive.

(2) The following conditions are equivalent:

(iv) For sufficiently large $n$, the group $\Gamma\left(L^{\otimes n}\right)$ has a basis consisting of strictly effective sections.

(v) Some positive power of $\bar{L}$ has a set of sections which has no base point and whose elements are strictly effective.

(vi) The height function $h_{L}$ has positive lower bound on $X$.

Proof. (i) $\rightarrow$ (ii): If a positive power $\bar{L}^{\otimes n}$ has a nontrivial strictly effective section $l$, then $h_{L}(x) \geq-\frac{1}{n} \log \|l\|$ when $l(x) \neq 0$. 
(ii) $\rightarrow$ (iii): (ii) implies that $e_{1}(\bar{L})>0$. By (5.2), we have $c_{1}(\bar{L})^{d}>0$, since by assumption $e_{d}(\bar{L}) \geq 0$.

(iii) $\rightarrow$ (i): By (1.9).

(iv) $\rightarrow$ (v): Trivial.

$(\mathrm{v}) \rightarrow(\mathrm{vi})$ : If a positive $\bar{L}^{\otimes n}$ has sections $\left\{l_{1}, \cdots, l_{k}\right\}$ such that $\cap \operatorname{div} l_{i}=\varnothing$ and $\left\|l_{i}\right\|<1$, then $e_{d}(\bar{L}) \geq-\max _{i} \log \left\|l_{i}\right\|>0$.

(vi) $\rightarrow$ (iv): Assume (vi). For any subvariety $Y$ of $X$ which is flat over spec $\mathbb{Z}$, applying $(5.2)$ to $Y$ we obtain that $c_{1}\left(\left.\bar{L}\right|_{Y}\right)^{\operatorname{dim} Y}>0$. The assertion follows from (4.2).

(5.8) Remark. It is a interesting question to understand the relations between numbers $e_{1}(\bar{L}), e_{2}(\bar{L}), \cdots, e_{d}(\bar{L})$. In the next section we will characterize torsion subvarieties of a multiplicative group using these numbers with respect to some canonical hermitian line bundles.

\section{Positivity of NAive heights}

(6.1) Let us recall the definition of a canonical height function on $\mathbb{P}^{n}(\overline{\mathbb{Q}})$. For each place $p$ of $\mathbb{Q}$, let $|\cdot|_{p}$ denote the valuation on $\mathbb{Q}$ such that $|p|_{p}=$ $p^{-1}$ if $p$ is finite, and let $|\cdot|_{\infty}$ denote the usual absolute value on $\mathbb{Q}$. Let $\mathbb{Q}_{p}$ denote the completion of $\mathbb{Q}$ with respect to $|\cdot|_{p}$, and let $\overline{\mathbb{Q}}_{p}$ denote an algebraic closure of $\mathbb{Q}_{p}$. The height function $h_{\max }$ is defined as follows. For a point $x=\left(x_{0}, x_{1}, \cdots, x_{n}\right)$ in $\mathbb{P}^{n}(\mathbb{Q})$, let $K$ denote the Galois closure of $\mathbb{Q}\left(x_{0}, \cdots, x_{n}\right)$; then we define

$$
h_{\max }(x)=\frac{1}{[K: \mathbb{Q}]} \sum_{p} \sum_{\sigma: K \rightarrow \mathbb{Q}_{p}} \log \max _{i}\left|\sigma x_{i}\right|_{p} .
$$

Consider $\mathbb{G}_{m}^{n}$ as the open subset $\left\{x_{0} x_{1} \cdots x_{n} \neq 0\right\}$ of $\mathbb{P}^{n}$. It is easy to see that $h_{\max }(x) \geq 0$ for any $x \in \mathbb{G}_{m}^{n}(\mathbb{Q})$, and $h_{\max }(x)=0$ if and only if $x$ is a torsion point of $\mathbb{G}_{m}^{n}(\overline{\mathbb{Q}})$. The main result of this section is the following theorem:

Theorem (6.2). Let $X$ be an irreducible subvariety of $\mathbb{G}_{m}^{n}$ over $\mathbb{Q}$. The following two statements are equivalent:

(1) For any nonempty open subset $U$, we have

$$
e_{U}=\inf _{x \in U} h_{\max }(x)=0 .
$$

(2) $X$ is a torsion subvariety of $\mathbb{G}_{m}^{n}$. This means that $X$ can be written as $x \cdot H$ in $\mathbb{G}_{m}^{n}$, where $x$ is a torsion point, and $H$ is a subgroup.

(6.3) Remarks. (1) The assertion of the above theorem does not change if we replace $h_{\max }$ by a height function $h$ on $\mathbb{G}_{m}^{n}(\mathbb{Q})=\mathbb{Q}^{* n}$ with property that there are two positive constants $c_{1}$ and $c_{2}$ such that for any $x$ in $\mathbb{G}_{m}(\overline{\mathbb{Q}})$, $c_{1} h_{\max }(x) \leq h(x) \leq c_{2} h_{\max }(x)$.

(2) Let $X$ be a subvariety of $\mathbb{G}_{m}^{n}$ defined over $\mathbb{Q}$. We say a torsion subvariety $W$ of $X$ is maximal if it is not contained in any larger torsion subvariety of $X$. Then (6.2) implies the following two assertions: (i) $X$ has only finitely many maximal torsion subvarieties $W_{1}, \cdots, W_{k}$; (ii) The height function $h_{\max }$ has a 
positive lower bound on $X-\cup W_{i}$. The assertion (i) is a theorem of Ihara, Serre, and Tate (see $\S 8.6$ of [Lan] ) when $\operatorname{dim} X=1$, and is a theorem of Laurent [Lau] and Sarnak [Sa] if $\operatorname{dim} X>1$. The assertion (ii) is an analogue of Lehmer's conjecture which claims that $h_{\max }(x)$ is bounded below by $c /[\mathbb{Q}(x): \mathbb{Q}]$ for any nontorsion point $x$ in $\overline{\mathbb{Q}}^{*}$, where $c$ is a positive constant.

(6.4) We will prove (6.2) using intersection theory. Fix a free group $V=$ $\mathbb{Z} u+\mathbb{Z} v$ of rank 2 . Let $\mathbb{P}^{1}$ denote the projective space associated to $V$; then $u, v$ can be considered as homogeneous coordinates of $\mathbb{P}^{1}$, and $V$ can be considered as space of sections of $O(1)$. We define a hermitian metric $\|\cdot\|_{\infty}$ as follows. For any point $x$ in $\mathbb{P}^{1}(\mathbb{C})$ and any local section $s$ of $O(1)$ near $x$ such that $s(x) \neq 0$,

$$
\|s\|_{\infty}=1 / \max \left(\left|\frac{u(x)}{s(x)}\right|,\left|\frac{v(x)}{s(x)}\right|\right) .
$$

Let $\bar{O}_{\infty}(1)$ denote the hermitian line bundle $\left(O(1),\|\cdot\|_{\infty}\right)$. For a positive integer $n$, let $\mathbb{P}_{n}$ denote the scheme $\left(\mathbb{P}^{1}\right)^{n}$, and also let $\bar{O}_{\infty}(1)$ denote the hermitian line bundle $\otimes_{i} \pi_{i}^{*} \bar{O}_{\infty}(1)$, where $\pi_{i}$ is the $i$-th projection from $\mathbb{P}_{n}$ to $\mathbb{P}^{1}$. Let $h_{\infty}$ denote the height function induced by $\bar{O}_{\infty}(1)$ on $\mathbb{P}_{n}(\overline{\mathbb{Q}})$. Consider $\mathbb{G}_{m}^{n}$ as the open subscheme of the generic fiber of $\mathbb{P}_{n}$ defined as the complement of $\left\{u_{1} v_{1} u_{2} v_{2} \cdots u_{n} v_{n}=0\right\}$, where $u_{i}=u \circ \pi_{i}$ and $v_{i}=v \circ \pi_{i}$. Then over $\mathbb{G}_{m}^{n}(\overline{\mathbb{Q}})$ we have $h_{\max } \leq h_{\infty} \leq n h_{\max }$.

We want to define heights for arithmetic subvarieties of $\mathbb{P}_{n}$ with respect to $\bar{O}_{\infty}(1)$. Notice that, on $\mathbb{P}^{1}(\mathbb{C})$, the metric $\|\cdot\|_{\infty}$ is not smooth, but it is the limit of $\left\{\|\cdot\|_{l}, l=1,2, \cdots\right\}$, where for each $l \geq 1,\|\cdot\|_{l}$ is defined as

$$
\|s\|_{l}(x)=\left(\left|\frac{u}{s}\right|^{l}(x)+\left|\frac{v}{s}\right|^{l}(x)\right)^{-1 / l} .
$$

Let $\bar{O}_{l}(1)$ denote the corresponding hermitian line bundle. We define heights with respect to $\bar{O}_{\infty}(1)$ as limits of heights with respect to $\bar{O}_{l}(1)$ by the following lemma.

The curvature $c_{1}^{\prime}\left(\bar{O}_{l}(1)\right)$ as a measure is given locally as

$$
\frac{\partial \bar{\partial}}{\pi i} \log \|s\|_{l}=-\frac{1}{l} \frac{\partial \bar{\partial}}{\pi i} \log \left(1+|z|^{l}\right)=\frac{d t d\left(\rho^{l}\right)}{\left(1+\rho^{l}\right)^{2}},
$$

where $z=v / u=\rho e^{2 \pi i t}$. It follows that for any continuous function $f$,

$$
\int f c_{1}^{\prime}\left(\bar{O}_{l}(1)\right)=\int_{0}^{\infty}\left[\int_{0}^{1} f\left(\rho^{1 / l} e^{2 \pi i t}\right) d t\right] \frac{d \rho}{(1+\rho)^{2}}
$$

Let $T$ denote the unit circle $\{(u, v):|u / v|=1\}$; then $\lim _{l \rightarrow \infty} c_{1}^{\prime}\left(\bar{O}_{l}(1)\right)=\delta_{T}$. Let $\bar{O}_{l}(1)$ also denote the hermitian line bundle $\sum_{i} \pi^{*} \bar{O}_{l}(1)$ on $\mathbb{P}_{n}$. Then $\bar{O}_{l}(1)$ has positive curvature. It follows that $\bar{O}_{l}(1)$ is semiample metrized. The following lemma gives some justifications for working on line bundles with limit metrics. 
Lemma (6.5). Let $X$ be an arithmetic variety of dimension $d$. Let $L$ be an ample line bundle and $L_{1}, \cdots, L_{d}$ be line bundles which have nonnegative degrees on any curves in any fibers.

(1) On each $L_{i}$, let $\|\cdot\|_{i}$ and $\|\cdot\|_{i}^{\prime}$ be two semipositive smooth metrics, and let $g_{i}=\log \|\cdot\|_{i}^{\prime}, \bar{L}_{i}=\left(L_{i},\|\cdot\|_{i}\right), \bar{L}_{i}^{\prime}=\left(L_{i},\|\cdot\|_{i}^{\prime}\right)$. Then for any nonzero rational section $s$ of $L_{d}$, one has

$$
\begin{aligned}
\left|\int_{X(\mathbf{C})} \log \|s\|_{d}^{\prime} c_{1}^{\prime}\left(\bar{L}_{1}^{\prime}\right) \cdots c_{1}^{\prime}\left(\bar{L}_{d-1}^{\prime}\right)-\int_{X(\mathbf{C})} \log \|s\|_{d} c_{1}^{\prime}\left(\bar{L}_{1}\right) \cdots c_{1}^{\prime}\left(\bar{L}_{d-1}\right)\right| \\
\leq \sum_{i=1}^{d}\left\|g_{i}\right\|_{\mathrm{sup}} c_{1}\left(L_{1, \mathbb{Q}}\right) \cdots c_{1}\left(L_{i-1, \mathbb{Q}}\right) c_{1}\left(L_{i+1, \mathbb{Q}}\right) \cdots c_{1}\left(L_{d, \mathbb{Q}}\right) \\
\quad+\sum_{i=1}^{d-1}\left\|g_{i}\right\|_{\text {sup }} c_{1}\left(L_{1, \mathbb{Q}}\right) \cdots c_{1}\left(L_{i-1, \mathbb{Q}}\right) c_{1}\left(L_{i+1, \mathbb{Q}}\right) \cdots c_{1}\left(L_{d-1, \mathbb{Q}}\right)|\operatorname{div}(s)|_{\mathbb{Q}},
\end{aligned}
$$

where if $\operatorname{div}(s)=\sum n_{i} Z_{i}$ with $Z_{i}$ integral, then $|\operatorname{div}(s)|=\sum\left|n_{i}\right| Z_{i}$.

(2) On each $L_{i}$, let $\|\cdot\|_{i}$ be a continuous metric and $\left\{\|\cdot\|_{i n}, n=1,2, \cdots\right\}$ be a sequence of smooth and semipositive metrics such that $\log \frac{\|\cdot\|_{i n}}{\|\cdot\|_{i}}$ converges uniformly to 0 . Let $\bar{L}_{\text {in }}=\left(L_{i},\|\cdot\|_{\text {in }}\right), \bar{L}_{i}=\left(L_{i},\|\cdot\|_{i}\right)$; then

$$
\lim _{n \rightarrow \infty} c_{1}\left(\bar{L}_{1 n}\right) \cdots c_{1}\left(\bar{L}_{d n}\right)
$$

exists and depends only on $\bar{L}_{1}, \cdots, \bar{L}_{d}$. We let $c_{1}\left(\bar{L}_{1}\right) \cdots c_{1}\left(\bar{L}_{d}\right)$ denote this limit.

(3) Let $\|\cdot\|$ be a continuous metric on $L$ which is the limit of smooth and semiample metrics. Let $\bar{L}=(L,\|\cdot\|)$; then

$$
d e_{1}(\bar{L}) \geq \frac{c_{1}(\bar{L})^{d}}{c_{1}\left(\bar{L}_{\mathbb{Q}}\right)^{d-1}} \geq e_{1}(\bar{L})+\cdots+e_{d}(\bar{L}) .
$$

Proof. For (1), one has

$$
\begin{aligned}
& \int_{X(\mathbb{C})} \log \|s\|_{d}^{\prime} c_{1}^{\prime}\left(\bar{L}_{1}^{\prime}\right) \cdots c_{1}^{\prime}\left(\bar{L}_{d-1}^{\prime}\right)-\int_{X(\mathbb{C})} \log \|s\|_{d} c_{1}^{\prime}\left(\bar{L}_{1}\right) \cdots c_{1}^{\prime}\left(\bar{L}_{d-1}\right) \\
& =\int_{X(\mathbb{C})}\left(\log \|s\|_{d}^{\prime}-\log \|s\|_{d}\right) c_{1}^{\prime}\left(\bar{L}_{1}^{\prime}\right) \cdots c_{1}^{\prime}\left(\bar{L}_{d-1}^{\prime}\right) \\
& \quad+\sum_{i=1}^{d-1} \int_{X(\mathbb{C})} \log \|s\|_{d} c_{1}^{\prime}\left(\bar{L}_{1}\right) \cdots c_{1}^{\prime}\left(\bar{L}_{i-1}\right)\left(c_{1}^{\prime}\left(\bar{L}_{i}^{\prime}\right)-c_{1}\left(\bar{L}_{i}\right)\right) c_{1}^{\prime}\left(\bar{L}_{i+1}^{\prime}\right) \cdots c_{1}^{\prime}\left(\bar{L}_{d-1}^{\prime}\right) \\
& =\int_{X(\mathbb{C})} g_{d} c_{1}^{\prime}\left(\bar{L}_{1}^{\prime}\right) \cdots c_{1}^{\prime}\left(\bar{L}_{d-1}^{\prime}\right) \\
& \quad+\sum_{i=1}^{d-1} \int_{X(\mathbb{C})} \log \|s\|_{d} c_{1}^{\prime}\left(\bar{L}_{1}\right) \cdots c_{1}^{\prime}\left(\bar{L}_{i-1}\right) \frac{\partial \bar{\partial}}{\pi i} g_{i} c_{1}^{\prime}\left(\bar{L}_{i+1}^{\prime}\right) \cdots c_{1}^{\prime}\left(\bar{L}_{d-1}^{\prime}\right),
\end{aligned}
$$

where $\partial, \bar{\partial}$ are in the distribution sense. Let $Z=\operatorname{div}(s)$; then

$$
\frac{\partial \bar{\partial}}{\pi i} \log \|s\|_{d}=c_{1}^{\prime}\left(\bar{L}_{d}\right)-\delta_{Z}
$$


and

$$
\begin{aligned}
\int_{X(\mathbb{C})} \log \|s\|_{d} c_{1}^{\prime}\left(\bar{L}_{1}\right) \cdots c_{1}^{\prime}\left(\bar{L}_{i-1}\right) \frac{\partial \bar{\partial}}{\pi i} g_{i} c_{1}^{\prime}\left(\bar{L}_{i+1}^{\prime}\right) \cdots c_{1}^{\prime}\left(\bar{L}_{d-1}^{\prime}\right) \\
=\int_{X(\mathbb{C})} g_{i} \frac{\partial \bar{\partial}}{\pi i} \log \|s\|_{d} c_{1}^{\prime}\left(\bar{L}_{1}\right) \cdots c_{1}^{\prime}\left(\bar{L}_{i-1}\right) c_{1}^{\prime}\left(\bar{L}_{i+1}^{\prime}\right) \cdots c_{1}^{\prime}\left(\bar{L}_{d-1}^{\prime}\right) \\
=\int_{X(\mathbb{C})} g_{i}\left(c_{1}^{\prime}\left(\bar{L}_{d}\right)-\delta_{Z}\right) c_{1}^{\prime}\left(\bar{L}_{1}\right) \cdots c_{1}^{\prime}\left(\bar{L}_{i-1}\right) c_{1}^{\prime}\left(\bar{L}_{i+1}^{\prime}\right) \cdots c_{1}^{\prime}\left(\bar{L}_{d-1}^{\prime}\right) .
\end{aligned}
$$

Since $c_{1}^{\prime}\left(\bar{L}_{i}\right)$ and $c_{1}^{\prime}\left(\bar{L}_{i}^{\prime}\right)$ are all semipositive, the inequality of (1) follows by replacing $g_{i}$ by $\left\|g_{i}\right\|_{\text {sup }}$ and $-\delta_{Z}$ by $\delta_{|Z|}$. then

For (2), let $s$ be a nonzero rational section of $L_{d}$, and let $\operatorname{div} s=\sum n_{i} Z_{i}$;

$$
\begin{aligned}
& c_{1}\left(\bar{L}_{1 n}\right) \cdots c_{1}\left(\bar{L}_{d n}\right)=c_{1}\left(\bar{L}_{1 n}\right) \cdots c_{1}\left(\bar{L}_{d-1, n}\right)\left(\sum n_{i} Z_{i},-\log \|s\|_{d n}\right) \\
& =\sum n_{i} c_{1}\left(\left.\bar{L}_{1 n}\right|_{Z_{i}}\right) \cdots c_{1}\left(\left.\bar{L}_{d-1, n}\right|_{Z_{i}}\right)-\int_{X(\mathbb{C})} \log \|s\|_{d n} c_{1}^{\prime}\left(\bar{L}_{1 n}\right) \cdots c_{1}^{\prime}\left(\bar{L}_{d-1, n}\right) .
\end{aligned}
$$

The assertion follows from (1) and induction on $\operatorname{dim} X$.

For (3), let $\left\{\|\cdot\|_{l}, l=1,2, \cdots\right\}$ be a sequence of smooth and semiample metrics on $L$ which is convergent uniformly to $\|\cdot\|$. Let $\bar{L}_{l}=\left(L,\|\cdot\|_{l}\right)$; by (5.2),

$$
d e_{1}\left(\bar{L}_{l}\right) \geq \frac{c_{1}\left(\bar{L}_{l}\right)^{d}}{c_{1}\left(\bar{L}_{l \mathbb{Q}}\right)^{d-1}} \geq e_{1}\left(\bar{L}_{l}\right)+\cdots+e_{d}\left(\bar{L}_{l}\right)
$$

Since $\log \frac{\|\cdot\|_{l}}{\|\cdot\|} \rightarrow 0$ as $l \rightarrow \infty$ uniformly, it follows $e_{i}\left(\bar{L}_{l}\right) \rightarrow e_{i}(\bar{L})$ and $c_{1}\left(\bar{L}_{l}\right)^{d} \rightarrow c_{1}(\bar{L})^{d}$. Letting $l \rightarrow \infty$ in the above inequalities, we obtain the inequality for $\bar{L}$.

Lemma (6.6). Let $X$ be an irreducible arithmetic hypersurface of $\mathbb{P}_{n}(n \geq 2)$ which is defined by a polynomial $F\left(x_{1}, \cdots, x_{n}\right)=\sum a_{i_{1}, \cdots, i_{n}} x_{1}^{i_{1}} \cdots x_{n}^{i_{n}}$ on $\mathbb{A}^{n}$ with property that if $a_{i_{1}, \cdots, i_{n}} \neq 0$ then $i_{2} \geq i_{1}$. Assume that $c_{1}\left(\left.\bar{O}_{\infty}(1)\right|_{X}\right)^{n}=0$. Then for any torsion point $\tau$ in $\overline{\mathbb{Q}}^{*}$ we have $c_{1}\left(\left.\bar{O}_{\infty}(1)\right|_{X_{\tau}}\right)^{n-1}=0$, where $X_{\tau}$ is an arithmetic subvariety of $X$ defined by the following polynomial over $\mathbb{Z}$ :

$$
\psi\left(x_{1}\right)=\prod_{\sigma: \mathbb{Q}(\tau) \rightarrow \mathbb{Q}}\left(u_{1}-\sigma(\tau) v_{1}\right) .
$$

Proof. First of all we have that $c_{1}\left(\bar{O}_{\infty}(1)\right)^{2}=0$ on $\mathbb{P}^{1}$. This follows from (6.5), (3). It follows that, for any subvariety $Y$ of $\mathbb{P}_{n}$ of dimension $d$,

$$
c_{1}\left(\left.\bar{O}_{\infty}(1)\right|_{Y}\right)^{d}=\sum_{i_{1}<i_{2}<\cdots<i_{d}} d ! c_{1}\left(\left.\pi_{i_{1}}^{*} \bar{O}_{\infty}(1)\right|_{Y}\right) \cdots c_{1}\left(\left.\pi_{i_{d}}^{*} \bar{O}_{\infty}(1)\right|_{Y}\right)
$$

Let $p_{i}$ denote the restriction of $\pi_{i}$ on $X$ and let $d=[\mathbb{Q}(\tau): \mathbb{Q}]$. By assumption $F$ is not contained in closed fibers of $\pi_{1}$, so $\psi$ is not a zero 
section of $p_{1}^{*}(O(d))$. Representing $d c_{1}\left(p_{1}^{*} \bar{O}_{l}(1)\right)$ by $\left(X_{\tau},-\log \|\psi\|_{l}\right)$, we have

$$
\begin{aligned}
0 & =d c_{1}\left(p_{1}^{*} \bar{O}_{\infty}(1)\right) \cdots c_{1}\left(p_{n}^{*} \bar{O}_{\infty}(1)\right) \\
& =\lim _{l \rightarrow \infty}\left(X_{\tau},-\log \|\psi\|_{l}\right) c_{1}\left(p_{2}^{*} \bar{O}_{l}(1)\right) \cdots c_{1}\left(p_{n}^{*} \bar{O}_{l}(1)\right) \\
& =\frac{1}{(n-1) !} c_{1}\left(\left.\bar{O}_{\infty}(1)\right|_{\left.X_{\tau}\right)}\right)^{n-1}-\lim _{l \rightarrow \infty} \int_{X(\mathbb{C})} \log \|\psi\|_{l} \delta_{l 2} \cdots \delta_{l n},
\end{aligned}
$$

where $\delta_{l i}=p_{i}^{*} c_{1}^{\prime}\left(\bar{O}_{l}(1)\right)$. To prove that $p_{1}^{-1}(\tau)$ has height 0 , it suffices to prove that

$$
A_{l X}=\int_{X} \log \|\psi\|_{l} \delta_{l 2} \cdots \delta_{l n} \text { has limit } 0 \text { as } l \rightarrow \infty .
$$

We want to use induction on $n$ to prove (6.6.1). It is trivial for $F=c x_{2}$ for some constant $c$. So we assume that $x_{2} \nmid F$. Now $F$ can be written as $x_{2} F_{1}+F_{2}$ with $F_{1}$ a polynomial of $\left(x_{1}, \cdots, x_{n}\right)$ and $F_{2}$ a nonzero polynomial of $\left(x_{3}, \cdots, x_{n}\right)$. It follows that $v_{2}$ does not vanish on any component of any fiber $p_{1}^{-1}(a)$ for $a \in \mathbb{C}$. Since

we obtain that

$$
\begin{gathered}
\delta_{l 2}=\frac{\partial \bar{\partial}}{\pi i} \log \left\|v_{2}\right\|_{l}+\delta_{p_{2}^{-1}(0)} \\
d \delta_{l 1}=\frac{\partial \bar{\partial}}{\pi i} \log \|\psi\|_{l}+\delta_{X_{\tau}}
\end{gathered}
$$

$$
\begin{aligned}
A_{l X} & =\int_{X(\mathbb{C})} \log \|\psi\|_{l} \frac{\partial \bar{\partial}}{\pi i} \log \left\|v_{2}\right\|_{l} \delta_{l 3} \cdots \delta_{l n}+\int_{p_{2}^{-1}(0)(\mathbb{C})} \log \|\psi\|_{l} \delta_{l 3} \cdots \delta_{l n} \\
& =\int_{X(\mathbb{C})} \log \left\|v_{2}\right\|_{l} \frac{\partial \bar{\partial}}{\pi i} \log \|\psi\|_{l} \delta_{l 3} \cdots \delta_{l n}+A_{l, p_{2}^{-1}(0)} \\
& =d \int_{X(\mathbb{C})} \log \left\|v_{2}\right\|_{l} \delta_{l 1} \delta_{l 3} \cdots \delta_{l n}-\int_{X_{\tau}(\mathbb{C})} \log \left\|v_{2}\right\|_{l} \delta_{l 3} \cdots \delta_{l n}+A_{l, p_{2}^{-1}(0)}
\end{aligned}
$$

We claim that

$$
c_{1}\left(\left.\bar{O}_{\infty}(1)\right|_{p_{2}^{-1}(0)}\right)^{n-1}=0 \text { and } \lim _{l \rightarrow \infty} \int_{X(\mathbb{C})} \log \left\|v_{2}\right\|_{l} \delta_{l 1} \delta_{l 3} \cdots \delta_{l n}=0
$$

Representing $c_{1}\left(p_{2}^{*} \bar{O}_{l}(1)\right)$ by $\left(p_{2}^{-1}(0),-\log \left\|v_{2}\right\|_{l}\right)$, we obtain that

$$
\begin{aligned}
0 & =c_{1}\left(p_{1}^{*} \bar{O}_{\infty}(1)\right) \cdots c_{1}\left(p_{n}^{*} \bar{O}_{\infty}(1)\right) \\
& =\lim _{l \rightarrow \infty}\left(p_{2}^{-1}(0),-\log \left\|v_{2}\right\|_{l}\right) c_{1}\left(p_{1}^{*} \bar{O}_{l}(1)\right) c_{1}\left(p_{3}^{*} \bar{O}_{l}(1)\right) \cdots c_{1}\left(p_{n}^{*} \bar{O}_{l}(1)\right) \\
& =\frac{1}{(n-1) !} c_{1}\left(\left.\bar{O}_{\infty}(1)\right|_{p_{2}^{-1}(0)}\right)^{n-1}-\lim _{l \rightarrow \infty} \int_{X(\mathbb{C})} \log \left\|v_{2}\right\|_{l} \delta_{l 1} \delta_{l 3} \cdots \delta_{l n} .
\end{aligned}
$$

The assertion (6.6.2) follows, since one can show that $c_{1}\left(\left.\bar{O}_{\infty}(1)\right|_{p_{2}^{-1}(0)}\right)^{n-1}$ is nonnegative by (6.5), (3), and that $\left\|v_{2}\right\|_{l} \leq 1$ by definition.

Since $p_{2}^{-1}(0)$ is defined by the equation $F_{2}=0$, one has that $p_{2}^{-1}(0) \subset$ $\pi_{2}^{-1}(0) \simeq \mathbb{P}_{n-1}$ satisfies the condition of the lemma. The assertion (6.6.1) for 
$n=2$ follows by applying (6.6.2) and the fact $p^{-1}(0)=\varnothing$ to the displayed formula for $A_{l X}$. For $n>2$, by induction one has that $\lim _{l \rightarrow \infty} A_{l p_{2}^{-1}(0)}=0$. Combining with $(6.6 .2)$, one has

$$
\lim _{l \rightarrow \infty} A_{l X}=-\lim _{l \rightarrow \infty} \int_{X_{\tau}(\mathbb{C})} \log \left\|v_{2}\right\|_{l} \delta_{l 3} \cdots \delta_{l n} .
$$

For any $x \in \mathbb{C}$ let $f_{l}(x)=\int_{p_{1}^{-1}(x)} \log \left\|v_{2}\right\|_{l} \delta_{l 3} \cdots \delta_{l n}$. Then $f_{l}(x)$ is a nonpositive pointwise continuous function on $\mathbb{C}$. By (6.5), (1), as $l \rightarrow \infty$, this $f_{l}$ converges uniformly. Let $f_{\infty}$ denote the limit; then $f_{\infty}$ must be a nonpositive pointwise continuous function. From (6.6.2) one has

$$
0=\lim _{l \rightarrow \infty} \int_{X(\mathbb{C})} \log \left\|v_{2}\right\|_{l} \delta_{l 1} \delta_{l 3} \cdots \delta_{l n}=\lim _{l \rightarrow \infty} \int_{\mathbb{C}} f_{l} c_{1}^{\prime}\left(\bar{O}_{l}(1)\right)=\int_{\mathbb{C}} f_{\infty} \delta_{T} .
$$

It follows that $f_{\infty}(x)=0$ for $x \in T$. By (6.6.3), $\lim _{l \rightarrow \infty} A_{l X}=0$. This proves (6.6.1) and therefore completes the proof of the lemma.

(6.7) Proof of (6.2). (2) $\rightarrow$ (1) is trivial, since the set of torsion points is Zariski dense in a torsion subvariety.

First of all we reduce the proof of $(1) \rightarrow(2)$ to the case that $X$ is a hypersurface of $\mathbb{G}_{m}^{n}$. Assume (6.2) is true for all hypersurfaces of all multiplicative groups. Let $X$ be a subvariety of $\mathbb{G}_{m}^{n}$ which satisfies (1) of (6.2) and $d=\operatorname{dim} X<n-1$. There is a projection $\pi$ from $\mathbb{G}_{m}^{n}$ to a factor $\mathbb{G}_{m}^{d+1}$ of the product of $d+1$ components of $\mathbb{G}_{m}^{n}$ such that $\pi X$ is a hypersurface of $\mathbb{G}_{m}^{d+1}$. It is easy to verify that $\pi X$ also satisfies (1) of (6.2). By assumption $\pi X$ can be written as $x H$, where $x$ is a torsion point of $\mathbb{G}_{m}^{d+1}$ and $H$ is a subgroup of $\mathbb{G}_{m}^{d+1}$. Replacing $X$ by $x^{-1} X$ (the assertions are invariant), we may assume that $x=1$, i.e., $\pi X$ is a subgroup. There is an isomorphism $p: \pi^{-1}(\pi X) \rightarrow \mathbb{G}_{m}^{n-1}$ of groups induced by a change of coordinates in $\mathbb{G}_{m}^{n}$. One can prove that $h_{\max }$ and $p^{*} h_{\max }$ are in the same equivalent class, as defined in (6.3). This shows that $p X$ satisfies (2) and therefore is a torsion subvariety by induction on $n$, so is $X$.

Now let $X$ be a hypersurface of $\mathbb{G}_{m}^{n}$ which satisfies (1) of (6.2). We want to prove the assertion (2) for $X$ using induction on $n$. If $n=1$ this is trivial, since $X$ must be a torsion point. We assume that $n \geq 2$. Let $X$ be defined by the equation $F=0$ where

$$
F=\sum_{i_{1} \geq 0, \cdots, i_{n} \geq 0} a_{i_{1}, \cdots, i_{n}} x_{1}^{i_{1}} \cdots x_{n}^{i_{n}}
$$

is an irreducible polynomial. Let $x_{2}^{k}$ be the highest power of $x_{2}$ dividing $F\left(0, x_{2}, \cdots, x_{n}\right)$. Changing coordinates $x_{1} \rightarrow x_{1} x_{2}^{k+1}, X$ is defined by the equation

$$
x_{2}^{-k} F\left(x_{1} x_{2}^{k+1}, x_{2}, \cdots, x_{n}\right)=0 \text {. }
$$

It is not difficult to prove that $x_{2}^{-k} F\left(x_{1} x_{2}^{k+1}, x_{2}, \cdots, x_{n}\right)$ is an irreducible polynomial. So by changing coordinates we may assume that $a_{i_{1}}, \cdots, i_{n} \neq 0$ 
implies that $i_{2} \geq i_{1}$. We claim that for any torsion point $\tau$ in $\mathbb{G}_{m}(\mathbb{Q})$ the preimage $\pi_{1}^{-1}(\tau) \cap X$ is a torsion subvariety.

Consider $\mathbb{G}_{m}^{n}$ to be an open subscheme that is the complement of $\left\{u_{1} v_{1} \cdots u_{n} v_{n}=0\right\}$, and let $\bar{X}$ denote the Zariski closure of $X$ in $\mathbb{P}_{n}$. By (6.5) we have that $c_{1}\left(\left.\bar{O}_{\infty}(1)\right|_{X}\right)^{n}=0$. By Lemma (6.6), for any torsion point $\tau \in \mathbb{G}_{m}(\mathbb{Q})$, the arithmetic subvariety $\bar{X}_{\tau}$ has height 0 . The geometric generic fiber of $\bar{X}_{\tau}$ over $\mathbb{Q}$ can be written as a union of $p_{1}^{-1}(\tau)=\{\tau\} \times Y$ and its conjugates in $\mathbb{Q}$, where $Y$ is a hypersurface $Y$ in $\mathbb{G}_{m}^{n-1}$. By $(6.5), p_{1}^{-1}(\tau)$ satisfies condition (1); so does $Y$, since $\tau$ is a torsion point. By induction, $Y$ is a torsion subvariety in $\mathbb{G}_{m}^{n-1}$, so is $p_{1}^{-1}(\tau)$ in $\mathbb{G}_{m}^{n}$. This proves our claim.

Write

$$
F\left(x_{1}, y\right)=\sum_{m} a_{m}\left(x_{1}\right) y^{m},
$$

where $m=\left(m_{2}, \cdots, m_{n}\right), y=\left(x_{2}, \cdots, x_{n}\right), a_{m}\left(x_{1}\right)$ are polynomials of $x_{1}$, and $y^{m}=x_{2}^{m_{2}} \cdots x_{n}^{m_{n}}$. Then by our claim if $x_{1}=\tau$ is a root of unity, then every irreducible component of the variety $X_{\tau}$ in $\mathbb{G}_{m}^{n-1}$ defined by $F_{\tau}=F(\tau, y)$ is torsion. Since torsion points in $\mathbb{G}_{m}$ are Zariski dense, there are infinitely many $\tau$ such that $X_{\tau}$ is irreducible and nonempty. In this case there are $m_{1}(\tau)$ and $m_{2}(\tau)$ such that

$$
F_{\tau}(y)=a_{m_{1}(\tau)}(\tau) y^{m_{1}(\tau)}+a_{m_{2}(\tau)}(\tau) y^{m_{2}(\tau)},
$$

where $a_{m_{1}(\tau)}(\tau)$ and $a_{m_{2}(\tau)}(\tau)$ are not zero and their ratio is a root of unity. Since there are only finitely many such pairs $\left(m_{1}(\tau), m_{2}(\tau)\right)$, one can find a pair $\left(m_{1}, m_{2}\right)$ such that $m_{1}(\tau)=m_{1}$ and $m_{2}(\tau)=m_{2}$ are true for infinitely many roots $\tau$ of unity. Let $\phi(x)=\frac{a_{m_{2}}(x)}{a_{m_{1}}(x)}$ as a rational map from $\mathbb{G}_{m}$ to $\mathbb{G}_{m}$, and let $\Gamma$ denote the graph of $\phi$ in $\mathbb{G}_{m}^{2}$. Then $\Gamma$ has infinitely many torsion points. By a theorem of Ihara, Serre, and Tate, $\Gamma$ must be a torsion subvariety of $\mathbb{G}_{m}^{2}$. It follows that $\phi(x)=a x^{n}$, where $a$ is a root of unity and $n$ is an integer. Without loss of generality, we may assume that $n \geq 0$; otherwise we interchange $m_{1}$ and $m_{2}$.

Let $\tilde{F}\left(x_{1}, y\right)=y^{m_{1}}+a x_{1}^{n} y^{m_{2}}$ and let $\tilde{X}$ denote the torsion subvariety of $\mathbb{G}_{m}^{n}$ defined by $\tilde{F}$. Then $\tilde{X} \cap X$ has dimension $\geq n-1$. It follows that $X$ is an irreducible component of $\tilde{X}$. So $X$ is a torsion subvariety of $\mathbb{G}_{m}^{n}$. This completes the proof of the theorem.

\section{APPENDIX: COHERENT SHEAVES WITH BOUNDED METRICS}

(a.1) Let $K$ be an algebraically closed valuation field. We assume that either $K$ is the archimedean field $\mathbb{C}$, or a nonarchimedean field which is an algebraic extension of a complete discrete valuation subfield $K_{0}$, with an algebraically closed residue field $k$. The valuation on $K$ is chosen such that each uniformizer of $K_{0}$ has valuation $e^{-1}$.

Let $V$ be a finite-dimensional vector space over $K$. A function $\|\cdot\|: V \rightarrow \mathbb{R}$ is called a $K$ norm if the following conditions are verified: 
(1) $\|k x\|=|k|\|x\|$;

(2) $\|x\| \geq 0$, and $\|x\|=0$ iff $x=0$;

(3) if $K$ is archimedean, then $\|x+y\| \leq\|x\|+\|y\|$, and if $K$ is nonarchimedean, then $\|x+y\| \leq \max (\|x\|,\|y\|)$.

Let $X$ be a projective scheme on $\operatorname{spec} K$, and let $F$ be a coherent sheaf on $X$. By a $K$-metric $\|\cdot\|$ on $F$ we mean a collection of $K$-norms on each fiber $F(x), x \in X(K)$.

Two metrics $\|\cdot\|$ and $\|\cdot\|^{\prime}$ are said to be in the same bounded class if the number

is finite.

$$
\sup _{x \in X(K), f \in F(x)-\{0\}}\left|\log \|f\|-\log \|f\|^{\prime}\right|
$$

(a.2) Let $L$ be a line bundle on $X$. Sometimes, we need to consider the "good" metrics. If $K=\mathbb{C}$, this means that the metrics are continuous on $X(K)$. If $K$ is nonarchimedean, this means that the metrics are "algebraic" as described as follows.

Let $E$ be a finite extension of $K_{0}$ in $K$, and let $R_{E}$ denote the valuation ring of $E$. Let $\tilde{X}$ be a projective variety on $\operatorname{spec} R_{E}$ with an isomorphism $\alpha: X \rightarrow \tilde{X}_{K}=\tilde{X} \times_{\operatorname{spec} R_{E}} \operatorname{spec} K$. Let $\tilde{L}$ be a line bundle $\tilde{L}$ on $\tilde{X}$ with an $\alpha$-isomorphism $\phi: L \rightarrow \tilde{L}_{K}=\tilde{L} \otimes_{R_{E}} K$. Then we can define a metric $\|\cdot\|_{\tilde{L}}$ on $L$ as follows. Via $\alpha$ and $\phi$ we may identify $X$ and $L$ with $\tilde{X}_{K}$ and $\tilde{L}_{K}$. Let $x: \operatorname{spec} K \rightarrow X$ be any algebraic point, and let $E^{\prime}$ denote the field $E(x)$; then $x$ can be factored through a $R_{E}$ morphism $\tilde{x}: \operatorname{spec} R_{E^{\prime}} \rightarrow \tilde{X}$. One has that $x^{*}(L) \simeq \tilde{x}^{*}(\tilde{L}) \otimes_{R_{E^{\prime}}} K$. For any $l \in x^{*}(L)$, we define

$$
\|l\|_{\tilde{L}}=\inf _{a \in K}\left\{|a|: l \in a \tilde{x}^{*}(L)\right\} .
$$

We say that the metrized line bundle $\bar{L}=\left(L,\|\cdot\|_{\tilde{L}}\right)$ is algebraic and is induced by the model $(\tilde{X}, \tilde{L})$.

Notice that any two "good" metrics on $L$ are in the same bounded class. So this bounded class depends only on $L$. We call any metric in this class a bounded metric. For any bounded metric $\|\cdot\|$, each section $l$ of $L$ on $X$ has a finite supremum norm $\|l\|_{\text {sup }}=\sup _{x \in X(K)}\|l\|(x)$. If $K$ is nonarchimedean and $\|\cdot\|$ is induced by a model $(\tilde{X}, \tilde{L})$, then $\|\cdot\|_{\text {sup }}$ is induced by $R_{E}$ module $\Gamma(\tilde{L})$ as follows. For $l \in \Gamma(L)$,

$$
\|l\|_{\text {sup }}=\inf _{a \in K}\left\{|a|: l \in a \Gamma(\tilde{L}) \otimes_{R_{E}} R_{K}\right\} .
$$

(a.3) For any coherent sheaf $F$, let $\phi_{F}: P_{F} \rightarrow X$ denote projective scheme $\operatorname{proj}_{X}(\operatorname{sym} F)$ over $X$ associated to $F$ and let $L_{F}$ denote the $O(1)$ bundle on $P_{F}$. Let $\|\cdot\|$ be a bounded metric on $L_{F}$. It induces a metric $\phi_{F *}\|\cdot\|$ on $F$ as follows: for any $x \in X(K)$ and any $f \in F(x)$ which we consider as a section of $L_{F}$ on $\phi^{-1}(x)$,

$$
\phi_{F *}\|f\|=\sup _{p \in \phi^{-1}(x)}\|f(p)\| .
$$

Notice that the bounded class of $\phi_{F *}\|\cdot\|$ does not depend on the choice of bounded metric $\|\cdot\|$; we call any metric in this class a bounded metric of $F$. 
Theorem (a.4). Let $\bar{F}=(F,\|\cdot\|)$ and $\bar{G}=(G,\|\cdot\|)$ be two coherent sheaves with bounded metrics and let $h: F \rightarrow G$ be a morphism. The the norm

$$
\|h\|_{\text {sup }}=\sup _{x \in X(K), f \in F(x)-\{0\}} \frac{\|h(f)\|}{\|f\|}
$$

is finite.

Proof. Since the assertion does not depend on the choice of the bounded metrics, we may assume that the metrics on $F, G$ are induced from bounded metrized line bundles $\bar{L}_{F}=\left(L_{F},\|\cdot\|\right), \bar{L}_{G}=\left(L_{G},\|\cdot\|\right)$. On $P_{G}$ we have a composite morphism

$$
h^{\prime}: \phi_{G}^{*} F \otimes L_{G}^{-1} \rightarrow \phi_{G}^{*} G \otimes L_{G}^{-1} \rightarrow O_{P_{G}} .
$$

It is easy to see that $\left\|h^{\prime}\right\|_{\text {sup }}=\|h\|_{\text {sup }}$. Replacing $X, F, G$ by $P_{G}, \phi_{G}^{*} F, O_{P_{G}}$ we may assume that $G=O_{X}$. Let $I$ denote the image of $h$; then $h$ is decomposed into $h_{1}: F \rightarrow I$ and $h_{2}: I \rightarrow O_{X}$. Put a bounded metric on $I$. We need only prove that both $h_{1}$ and $h_{2}$ have finite norms.

Replacing $h: F \rightarrow G$ by $h_{1}: F \rightarrow I$ in the above paragraph, we may assume that $I=O_{X}$. This defines a morphism $j: X \rightarrow P_{F}$ and an isomorphism $h_{3}: j^{*} L_{F} \rightarrow O_{X}$ such that $h_{1}$ is the composition of $h_{3}$ and the canonical morphism $h_{4}: F \rightarrow j^{*} L_{F}$. Now $\left\|h_{4}\right\| \leq 1$ by definition, and $\left\|h_{3}\right\|$ is bounded since $h_{3}$ is an isomorphism of line bundles with bounded metrics. So $h_{1}$ has finite norm.

For $h_{2}$, let $\psi: B \rightarrow X$ denote the blow up of $X$ with respect to $I$; then $I O_{B}$ is an invertible ideal sheaf. The morphism $\psi^{*}\left(h_{2}\right)$ is decomposed into $h_{5}: \psi^{*} I \rightarrow I O_{B}$ and $h_{6}: I O_{B} \rightarrow O_{B}$. Put a bounded metric on $I O_{B}$. Now $h_{5}$ is surjective, and it has finite norm by the above paragraph. $h_{6}$ has finite norm since it is a morphism of two line bundles. This completes the proof of the theorem.

\section{REFERENCES}

[A] A. Arakelov, Intersection theory of divisors on an arithmetic surface, Izv. Akad. Nauk SSR Ser. Mat. 86 (1974), 1167-1180.

[BV] J. M. Bismut and E. Vasserot, The asymptotics of the Ray-Singer analytic torsion associated with higher powers of a positive line bundle, Comm. Math. Phys. 125 (1989), 355-367.

[D] P. Deligne, La determinant de la cohomologie, Contemp. Math., no. 67, Amer. Math. Soc., Providence, RI, 1987.

[F1] G. Faltings, Calculus on arithmetic surfaces, Ann. of Math. (2) 119 (1984), 387-424.

[F2] Lectures on the arithmetic Riemann-Roch theorem, Notes by S. Zhang, Ann. of Math. Stud., vol. 127, Princeton Univ. Press, Princeton, NJ, 1992.

[GS1] H. Gillet and C. Soulé, Arithmetic intersection theory, Inst. Hautes Études Sci. Publ. Math. 72 (1990), 94-174.

[GS2] __ An arithmetic Riemann-Roch theorem, Invent. Math. 110 (1992), 473-543.

[Ha] R. Hartshorne, Ample subvarieties of algebraic varieties, Lecture Notes in Math., vol. 156, Springer-Verlag, Berlin and New York, 1970.

[Hi] H. Hironaka, Resolution of singularities of an algebraic variety over a field of characteristic zero. I, II, Ann. of Math. (2) 79 (1964), 109-208. 
[Lan] S. Lang, Fundamentals of diophantine geometry, Springer-Verlag, Berlin and New York, 1983.

[Lau] M. Laurent, Equations diophantiennes exponentielles, Invent. Math. 78 (1984), 299-327.

[Sa] P. Sarnak, Betti numbers of congruence groups, Preprint.

[Sz] L. Szpiro, Sur les proprietes numeriques du dualisant-relatif d'une surface arithmetique, The Grothendieck Festschrift, Vol. 3, Birkhauser, Boston, 1990, pp. 229-246.

[T] G. Tian, On a set of polarized Kahler metrics on algebraic manifolds, J. Differential Geom. 32 (1990), 99-130.

[Z1] S. Zhang, Positive line bundles on arithmetic surfaces, Ann. of Math. (2) 136 (1992), 569-587.

[Z2] _ Admissible pairing on a curve, Invent. Math. 112 (1993), 171-193.

Department of Mathematics, Princeton University, Fine Hall, Washington Road, Princeton, New JeRsey 08544

E-mail address: szhang@ math.princeton.edu 